\title{
A new view of the Cygnus $X$ region
}

\section{KOSMA ${ }^{13} \mathrm{CO} 2 \rightarrow 1,3 \rightarrow 2$, and ${ }^{12} \mathrm{CO} 3 \rightarrow 2$ imaging ${ }^{\star}$}

\author{
N. Schneider ${ }^{3,2,1}$, S. Bontemps ${ }^{2}$, R. Simon ${ }^{1}$, H. Jakob ${ }^{1}$, F. Motte ${ }^{3}$, M. Miller ${ }^{1}$, C. Kramer ${ }^{1}$, and J. Stutzki ${ }^{1}$ \\ 1 I. Physikalisches Institut, Universität zu Köln, Zülpicher Straße 77, 50937 Köln, Germany \\ 2 Observatoire de Bordeaux, BP 89, 33270 Floirac, France \\ 3 SAp/CEA Saclay, 91191 Gif-sur-Yvette, France \\ e-mail: nicola.schneider-bontemps@cea.fr
}

Received 24 February 2006 / Accepted 28 June 2006

\section{ABSTRACT}

\begin{abstract}
Context. The Cygnus X region is one of the richest star formation sites in the Galaxy. There is a long-standing discussion about whether the region is a chance superposition of several complexes along the line of sight or a single coherent complex at a distance of 1.5 to $2 \mathrm{kpc}$.

Aims. Combining a ${ }^{13} \mathrm{CO} 2 \rightarrow 1$ survey taken with the KOSMA $3 \mathrm{~m}$ telescope with mid-IR images from MSX provides a way to improve our understanding of the spatial structure of the complex. The physical properties of the molecular gas can be derived in more detail as it was done in former studies.

Methods. Cygnus X has been mapped in ${ }^{13} \mathrm{CO} J=2 \rightarrow 1\left(10.8 \mathrm{deg}^{2}\right)$ at an angular resolution of $130^{\prime \prime}$, as well as for smaller areas in ${ }^{12} \mathrm{CO}$ and ${ }^{13} \mathrm{CO} J=3 \rightarrow 2\left(90^{\prime \prime}\right)$, using the KOSMA 3 m submm-telescope.

Results. We identified 91 clumps in ${ }^{13} \mathrm{CO} 2 \rightarrow 1$ that have a typical excitation temperature of $10-30 \mathrm{~K}$, an average density of $1.3 \times 10^{3} \mathrm{~cm}^{-3}$, radii of $1-8 \mathrm{pc}$, and masses of a few hundred to several ten thousand $M_{\odot}$. The main cloud complexes, the northern part $\left(M \simeq 2.8 \times 10^{5} M_{\odot}\right)$ including DR21 and W75N and the southern region $\left(M \simeq 4.5 \times 10^{5} M_{\odot}\right)$ with IC $1318 \mathrm{~b} / \mathrm{c}$ and AFGL2591, show differences in their physical properties. The ${ }^{13} \mathrm{CO}$ emission is closely associated with mid-IR emission seen with MSX. We find evidence that Cygnus OB2 and Cygnus OB9 are affecting the molecular material in Cygnus X.

Conclusions. Since essentially all molecular cloud complexes in Cygnus $\mathrm{X}$ form groups that are connected by molecular emission (visible in channel and position-velocity maps) and partly show evidence of interaction with UV radiation, we conclude that most of the objects seen in this region are located at the same distance, i.e., that of the $\mathrm{OB} 2$ cluster at $\sim 1.7 \mathrm{kpc}$, which is also consistent with the distances of other OB associations (OB9, OB1) in Cygnus X.
\end{abstract}

Key words. ISM: clouds - ISM: structure - ISM: individual objects: Cygnus X region - submillimeter

\section{Introduction}

It is well established that low- and high-mass stars form in cold $(<20 \mathrm{~K})$, dense $\left(n>10^{5} \mathrm{~cm}^{-3}\right)$ cores of (giant) molecular clouds (GMCs). While low-mass stars have no significant influence on their environment during and after their lifetimes, massive stars inject large quantities of energy into the interstellar medium via their radiation and stellar winds and ultimately as supernovae. These dynamical processes can trigger star formation due to the induced collapse of molecular cloud fragments at the edges of expanding $\mathrm{H}_{\text {II }}$ regions and supernova shells or to direct compression of globules (e.g., Elmegreen 1998). Accordingly, massive stars largely determine the evolution of surrounding clouds and can finally lead to their destruction.

Numerous studies of molecular clouds show that a cloud's physical state, characterized by properties such as temperature, mass, radius, and $\mathrm{H}_{2}$ (column) density regulate the efficiency of star formation. While diffuse, cold, low-column density clouds in the solar neighborhood only form isolated low-mass stars or show no star formation (Maddalena \& Thaddeus 1985), only GMCs with massive cores of dense molecular gas are able to form high-mass stars and clusters. It is thus of vital interest to

* Appendices are only available in electronic form at http://www . aanda. org study molecular gas properties over a wide range of environments in order to better understand the detailed processes related to star formation. In particular, complete surveys in isotopomeric low- $J$ CO lines of active star-forming regions that are not too far away $(<3 \mathrm{kpc})$ provide a good statistical evaluation of the properties of the lower-density gas. The cloud clumps identified in such surveys contain high-mass dense cores that actually form stars.

In order to investigate the massive star formation capacity of such a GMC and to derive the physical properties of the molecular environment of candidate high-mass protostellar objects and protostars, we selected the Cygnus $\mathrm{X}$ region for a comprehensive multiwavelength study. This paper presents an extended $\left(10.8 \mathrm{deg}^{2}\right)$ survey of the molecular gas in Cygnus $\mathrm{X}$ in the ${ }^{13} \mathrm{CO} J=2 \rightarrow 1$ line (selected regions are also covered in the ${ }^{13} \mathrm{CO}$ and ${ }^{12} \mathrm{CO} J=3 \rightarrow 2$ lines) obtained with the KOSMA $3 \mathrm{~m}$ submm-telescope. A $1.3 \mathrm{~mm}$ continuum survey using MAMBO at the IRAM $30 \mathrm{~m}$ telescope (Motte et al. 2005, 2006, in prep.) identifies the protostellar objects in Cygnus X, a global study of the infrared source population is given in Bontemps et al. (2006, in prep.), observations of PDR lines in the DR2 1 region are presented in Jakob et al. (2006), and a ${ }^{13} \mathrm{CO}, \mathrm{C}^{18} \mathrm{O} 1 \rightarrow 0$ and $\mathrm{N}_{2} \mathrm{H}^{+} 1 \rightarrow 0$, and CS $2 \rightarrow 1$ survey using the $\mathrm{FCRAO}^{1}$ is shown

\footnotetext{
${ }^{1}$ Five College Radio Astronomy Observatory.
} 
in Simon et al. (2006, in prep.) and Schneider et al. (2006, in prep.).

In the present paper, we focus mainly on the identification and description of clouds and clumps in position and velocity space from the observed, very complex CO data in combination with mid-IR emission. The ${ }^{13} \mathrm{CO}$ data are then used to measure the total mass of the clouds, as well as other parameters such as temperature, (column) density, and opacity. This analysis will yield a compilation of the global cloud properties in Cygnus $\mathrm{X}$ and, in addition, set the stage for cross correlation with other data sets and further analysis in upcoming papers.

The Cygnus X region is close to Galactic longitude $l=90^{\circ}$. Here, the local Galactic arm, the Perseus arm, and the outer Galaxy are found along the same line of sight, covering distances between 1 and $8 \mathrm{kpc}$. Using a standard Galactic rotation curve (e.g., Clemens 1985), the tangent point velocity in that direction is $3-4 \mathrm{~km} \mathrm{~s}^{-1}$ and the distance to the sun at the position of the tangent point is roughly $1.5 \mathrm{kpc}$. Since radial velocities around the tangent point in Cygnus $\mathrm{X}$ are close to zero, they do not provide reliable distances.

In this paper, we concentrate on the local arm (sometimes also called "Cygnus arm"), which contains the vast majority of $\mathrm{CO}$ gas detected in our survey (less than approximately $1 \%$ of the ${ }^{13} \mathrm{CO}$ emission is at velocities lower than $-20 \mathrm{~km} \mathrm{~s}^{-1}$, i.e., tracing material at larger distances beyond the tangent point). One of the goals of this paper is to suggest a solution for the distance problem by combining large-scale images of molecular lines with mid-infrared emission tracing local PDRs in the vicinity of massive stars.

A short review of what is presently known on Cygnus $\mathrm{X}$ as a whole region is presented in Sect. 2 in order to place our new data in context with previous observations. The observational parameters are given in Sect. 3 and the CO data displayed and described in Sect. 4. Overlays to mid-IR maps from $\mathrm{MSX}^{2}$ are shown and discussed in Sect. 5. Using the multiwavelength data shown in the sections before, we draw a new comprehensive scenario for the Cygnus X region in Sect. 6.

\section{Overview of the region}

The name Cygnus $X$ for this region was coined by Piddington $\&$ Minnet (1952) due to its strong and extended Galactic radio continuum emission. A large number of radio surveys cover the region (e.g., $1390 \mathrm{MHz}$, Westerhout 1958; $5 \mathrm{GHz}$, Downes \& Rinehart 1966; 408, 1420, and $4800 \mathrm{MHz}$, Wendker et al. 1984, 1991) and hundreds of thermal continuum sources, i.e., H II regions, superimposed on a non-thermal background, were detected. Several supernova remnants were identified (e.g., Uyaniker et al. 2001) including the well-studied source G78.2+2.1 ( $\gamma$-Cygni, e.g., Bykov et al. 2004), and a even larger number is required to explain the Cygnus superbubble scenario (Cash et al. 1980) concluded from X-ray observations.

Figure 1 (top) shows an $\mathrm{H}_{\alpha}$ image (courtesy of William McLaughlin $^{3}$ ) of the Galactic plane in the Cygnus X direction. In this optical image, the large-scale dark pattern known as the "Great Cygnus Rift" and the bright, well-known reflection nebulae North America nebula (NGC 7000), Pelican nebula (IC 5070), and IC 1318 are most prominent.

The global distribution of the total column density of gas and dust in the Cygnus $\mathrm{X}$ direction is shown in the bottom panel

\footnotetext{
2 Midcourse Space Experiment, Egan et al. (1998).

3 Willmclaughlin.astrodigitals.com
}

of Fig. 1. The column density, as traced by our 2MASS nearinfrared extinction map (see Sect. 3 for details), correlates well with the CO contours from low angular-resolution (8:7) surveys by Cong (1977), Dame et al. (1987), and Leung \& Thaddeus (1992). In this figure, the various known OB associations, the most prominent radio sources, and dark clouds are all indicated together with the area we have surveyed in the present work. Comparison of the two figures clearly shows that the Great Rift is actually rather thin and does not dominate the total column density of interstellar gas toward Cygnus X. The obscuration caused by the Rift, however, is high enough to make most of the radio continuum sources optically invisible or highly reddened.

The OB associations and young clusters in the region have been revisited recently in IR surveys (Knödlseder 2000; Comeron \& Torra 2001; Dutra \& Bica 2001; Le Duigou \& Knödlseder 2002). In particular, it was recognized that Cyg OB2 at $1.7 \mathrm{kpc}$ distance is a young, very rich association containing on the order of a hundred O-stars, making it the largest known association in the Galaxy outside the Galactic center. It could, therefore, be an equivalent of a proto-globular cluster (Knödlseder 2000). More recently, Comeron \& Pasquali (2006, in prep.) suggest that this exceptional association could be even richer in stars and spatially more extended by a factor of 2 .

The slightly older (Uyaniker et al. 2001) OB associations Cyg OB1, OB9, and OB8 are known to be at distances around 1.5 to $2 \mathrm{kpc}$ with typical uncertainties of 0.3 to $0.5 \mathrm{kpc}$. The age and distance of Cyg OB6 are uncertain, while the Cyg OB7 association lies at a distance of $0.7 \mathrm{kpc}$ (Uyaniker et al. 2001), which makes it part of Gould's Belt and associated with the Great Cygnus Rift and the North America and Pelican nebula complex (NGC 7000, IC 5070, L935, and L936). Apart from the known nearby objects (NGC 7000, IC 5070, the Great Rift, OB6, and OB7), the large-scale view of Cygnus X reveals two large molecular complexes apparently located on either side of Cyg OB2 at the heart of the Cygnus X complex. The older $\mathrm{OB}$ associations at lower longitudes are associated with much less molecular gas as expected for more evolved regions.

The Cygnus X region constitutes a large reservoir of molecular gas. From the ${ }^{12} \mathrm{CO} 1 \rightarrow 0 \mathrm{CfA}$ survey, we derived a total gas mass of $4.7 \times 10^{6} M_{\odot}$ within the area outlined in Fig. 1, assuming a common distance of $1.7 \mathrm{kpc}$. The mass was determined from the line integrated $\left(-10\right.$ to $\left.20 \mathrm{~km} \mathrm{~s}^{-1}\right){ }^{12} \mathrm{CO}$ intensity $I\left({ }^{12} \mathrm{CO}\right)$ inside the area of the lowest contour line and the conversion factor $X=N\left(\mathrm{H}_{2}\right) / I\left({ }^{12} \mathrm{CO}\right)=2.75 \times 10^{20}$ (Bloemen et al. 1986) to estimate the $N\left(\mathrm{H}_{2}\right)$ column density. A value of $4 \times 10^{6} M_{\odot}$ was estimated from our $A_{\mathrm{v}}$ map. Cygnus $\mathrm{X}$ is thus among the most massive GMC complexes in the Milky Way.

\section{Observations}

The ${ }^{12} \mathrm{CO}$ (or $\left.{ }^{13} \mathrm{CO}\right) 3 \rightarrow 2$ and ${ }^{13} \mathrm{CO} 2 \rightarrow 1$ lines were observed simultaneously during several observing periods between November 2001 and April 2005 at the Kölner Observatorium für Submm-Astronomie (KOSMA), which operates a $3 \mathrm{~m}$ submmtelescope on Gornergrat, Switzerland. The observations were performed with a dual-channel SIS-receiver, built at the Cologne Institute, operating at 210-270 and 325-365 GHz (Graf et al. 1998). Two acousto optical spectrometers of the Cologne group were used as backends (Schieder et al. 1989). The mean zenith opacities were 0.11 at $220 \mathrm{GHz}, 0.48$ at $330 \mathrm{GHz}$ and 0.30 at $345 \mathrm{GHz}$. The observations at $330 \mathrm{GHz}$ were corrected for sideband imbalance due to an atmospheric water line at $325 \mathrm{GHz}$, as derived from an atmospheric model of Grossman (1989). The spectra were calibrated to a main-beam brightness temperature 

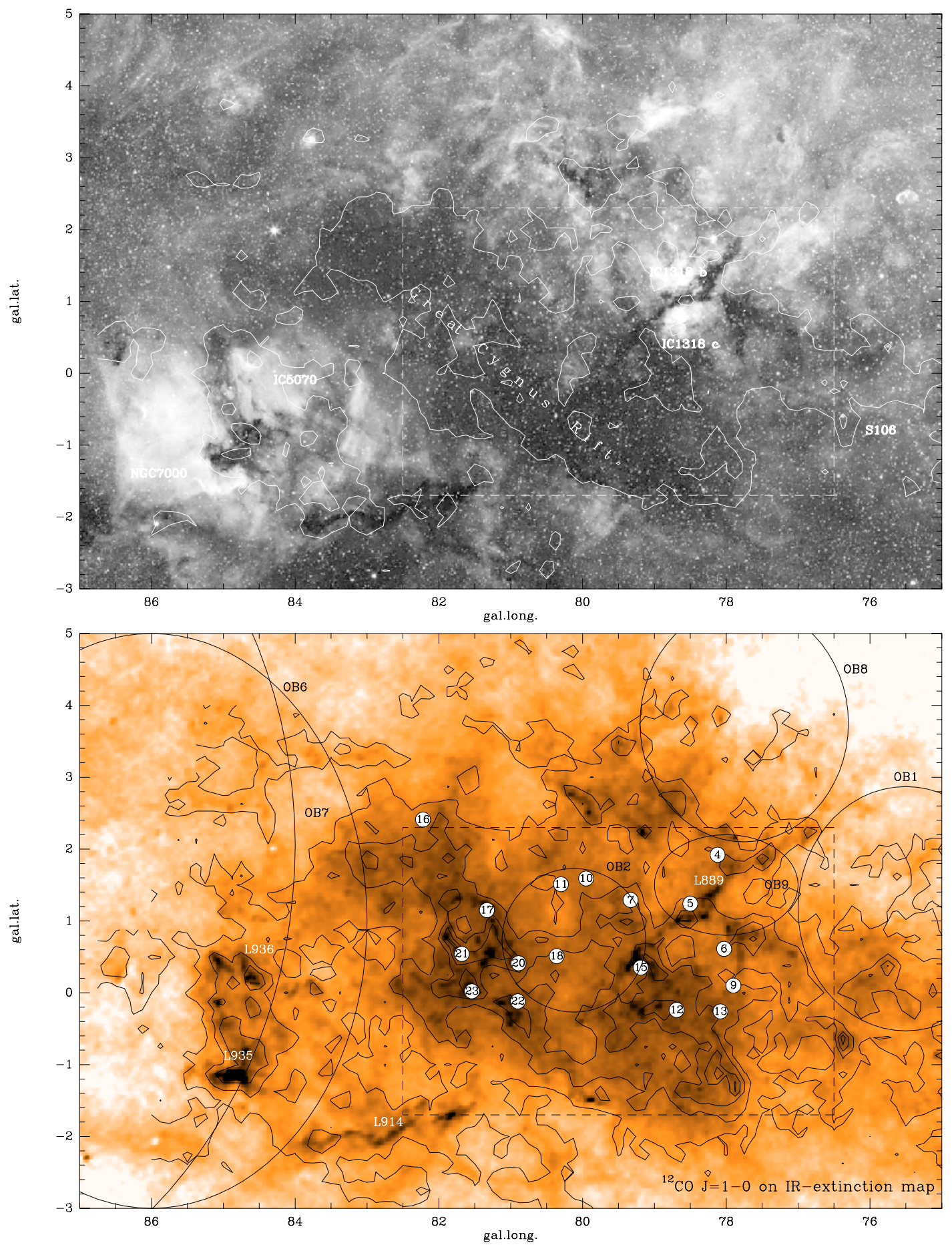

Fig. 1. Top: $\mathrm{H}_{\alpha}$ image (courtesy of William McLaughlin). Overlaid are white contours $\left(20 \%, 60 \%\right.$, and $80 \%$ ) of ${ }^{12} \mathrm{CO} 1 \rightarrow 0$ line emission from the CfA (Center for Astrophysics) survey (Dame et al. 1987). The prominent H II regions North America nebula NGC 7000 and Pelican nebula IC 5070 (together known as W80) and IC 1318 b/c are indicated. The large-scale extinction feature across the whole Cygnus X region ("the Great Rift") is designated as well. Bottom: extinction map obtained from near-infrared 2MASS data, expressed as visual extinction: $A_{\mathrm{v}}$ from 1 (white) to 32 (black) magnitudes. Overlaid are black contours ( $10 \%$ to $90 \%$ of maximum intensity $39.7 \mathrm{~K} \mathrm{~km} \mathrm{~s}^{-1}$ in steps of $\left.20 \%\right)$ of ${ }^{12} \mathrm{CO} 1 \rightarrow 0$ line integrated ( -10 to $20 \mathrm{~km} \mathrm{~s}^{-1}$ ) emission from the CfA survey. The black ellipses represent the extent of the OB clusters (taken from Uyaniker et al. 2001). Small numbered circles denote the positions of radio continuum sources (DR4 to 23) as identified by Downes \& Rinehart (1966). The area that will be shown in more detail in the mid-IR (Figs. 7 to 9), including the regions mapped with KOSMA, is marked by a black dashed rectangle. The largest Lynds (1962) dark clouds are indicated in white letters.

scale $\left(T_{\mathrm{mb}}\right)$ using the beam efficiencies listed in Table 1 . The forward efficiency was derived from skydips to be 0.9 .

Pointing was monitored simultaneously for both spectral channels using continuum cross scans of Jupiter and were found to be accurate to within $15^{\prime \prime}$. The offset between the two beams was derived in the same manner and found to be smaller than $30^{\prime \prime}$ in elevation. For efficient mapping, we used the on-the-fly (OTF) observing mode at KOSMA (Kramer et al. 1999). Two main regions were mapped on a $30^{\prime \prime}$ grid: an area of 3.3 square degrees $\left(\mathrm{deg}^{2}\right)$ around the radio sources DR17-DR23, which 
Table 1. Observational parameters of the data sets obtained with KOSMA. Columns one and two indicate the line and transition frequency, followed by the number of points, the spacing (the data on a sampling raster of $30^{\prime \prime}$ were regridded on a $60^{\prime \prime}$ raster), and half power beam width (HPBW) in arcsec; $\eta_{\mathrm{mb}}$ is the main-beam efficiency, $T_{\text {sys }}$ the average system temperature, $\Delta v_{\text {res }}$ denotes the channel spacing, and $\Delta T_{\text {rms }}$ the average rms noise temperature (of the data on a $60^{\prime \prime}$ grid) per channel on a $T_{\mathrm{mb}}$ scale.

\begin{tabular}{ccccccccc}
\hline \hline & $\begin{array}{c}v \\
{[\mathrm{GHz}]}\end{array}$ & \#Points & Grid & HPBW & $\eta_{\mathrm{mb}}$ & $\begin{array}{c}\left\langle T_{\text {sys }}\right\rangle \\
{[\mathrm{K}]}\end{array}$ & $\begin{array}{c}\Delta v_{\text {res }} \\
{\left[\mathrm{km} \mathrm{s}^{-1}\right]}\end{array}$ & $\begin{array}{c}\left\langle\Delta T_{\text {rms }}\right\rangle \\
{[\mathrm{K}]}\end{array}$ \\
\hline${ }^{13} \mathrm{CO} \mathrm{3 \rightarrow 2}$ & 330.588 & 9834 & $60^{\prime \prime}$ & $80^{\prime \prime}$ & 0.68 & 325 & 0.31 & 0.35 \\
${ }^{13} \mathrm{CO} \mathrm{2} \rightarrow 1$ & 220.399 & 37778 & $60^{\prime \prime}$ & $130^{\prime \prime}$ & 0.72 & 219 & 0.22 & 0.34 \\
${ }^{12} \mathrm{CO} \mathrm{3 \rightarrow 2}$ & 345.796 & 21934 & $60^{\prime \prime}$ & $80^{\prime \prime}$ & 0.68 & 346 & 0.29 & 0.38 \\
${ }^{12} \mathrm{CO} \mathrm{2} \rightarrow 1$ & 230.537 & 77 & & $130^{\prime \prime}$ & 0.72 & 222 & 0.21 & 0.09 \\
\hline
\end{tabular}

will be called the "CygX-North" region from now on, and an area of $7.5 \mathrm{deg}^{2}$ around DR4-DR15, which we refer to as "CygX-South". This division is similar to the choice of Cong (1977), who named the two complexes the DR21 region and the IC 1318 region. The map center position is $\mathrm{RA}(\mathrm{J} 2000.0)=$ $20^{\mathrm{h}} 28^{\mathrm{m}}$ and $\operatorname{Dec}(\mathrm{J} 2000.0)=39^{\circ} 30^{\prime}$. We performed scans at constant Declination with a length of $10^{\prime}$. An emission-free position at $\mathrm{RA}(\mathrm{J} 2000.0)=20^{\mathrm{h}} 37^{\mathrm{m}} 10.0^{\mathrm{s}}$ and $\operatorname{Dec}(\mathrm{J} 2000.0)=42^{\circ} 30^{\prime}$ was used as the off-position for the observations of CygX-North. For CygX-South, we used an OFF-position for $1 / 3$ of the area mapped at $\mathrm{RA}(\mathrm{J} 2000.0)=20^{\mathrm{h}} 27^{\mathrm{m}} 07.0^{\mathrm{s}}$ and $\operatorname{Dec}(\mathrm{J} 2000.0)=$ $39^{\circ} 58^{\prime}$, which shows a weak $(\sim 0.1 \mathrm{~K}){ }^{13} \mathrm{CO} 2 \rightarrow 1$ emission feature at $-8 \mathrm{~km} \mathrm{~s}^{-1}$. For the rest of the map, an emission-free position at $\mathrm{RA}(\mathrm{J} 2000.0)=20^{\mathrm{h}} 32^{\mathrm{m}} 17.0^{\mathrm{s}}$ and $\operatorname{Dec}(\mathrm{J} 2000.0)=41^{\circ} 41^{\prime}$ was used.

The ${ }^{13} \mathrm{CO} 3 \rightarrow 2$ map covers parts of CygX-North $\left(\sim 1.5 \mathrm{deg}^{2}\right)$, and CygX-South $\left(\sim 1 \mathrm{deg}^{2}\right)$ while the ${ }^{12} \mathrm{CO} 3 \rightarrow 2$ map focusses on the CygX-South region $\left(\sim 6 \mathrm{deg}^{2}\right)$. A total of 77 individual positions were observed in the ${ }^{12} \mathrm{CO} 2 \rightarrow 1$ line (see Table 1) with the same frontend and backend configuration. The spectra were taken on peaks of ${ }^{13} \mathrm{CO}$ emission and have a signal-to-noise ratio of typically better than 50 .

The extinction map of the Cygnus X region shown in Fig. 1 (bottom) is derived from the publicly available $2 \mathrm{MASS}^{4}$ point source catalog by calculating the average reddening of stars with a method adapted from those described in Lada et al. (1994), Lombardi \& Alves (2001), and Cambrésy et al. (2002). The extinction is derived from the reddening of both $[\mathrm{J}-\mathrm{H}]$ and $[\mathrm{H}-\mathrm{K}]$ colors. From the stellar population model by Robin et al. (2003), we obtained a predicted density of foreground stars for a distance to the complex of $1.7 \mathrm{kpc}$. For each $2^{\prime}$ size pixel of the map, this expected number of foreground stars is removed from the least reddened 2MASS sources before deriving the average reddening (see Bontemps et al. 2006, in prep., for details).

\section{Results: ${ }^{13} \mathrm{CO} 2 \rightarrow 1$ and ${ }^{12} \mathrm{CO}$ and ${ }^{13} \mathrm{CO} 3 \rightarrow 2$ mapping}

Figures 2, A.1, and A.2 show the line integrated (-10 to $20 \mathrm{~km} \mathrm{~s}^{-1}$ ) ${ }^{13} \mathrm{CO} 2 \rightarrow 1$ and ${ }^{13} \mathrm{CO}$ and ${ }^{12} \mathrm{CO} 3 \rightarrow$ 2 maps covering essentially all prominent high (column) density regions in the extinction map displayed in Fig. 1 around the radio sources DR4 to DR23. In the following, we discuss the $\mathrm{CygX}$-North and $\mathrm{CygX}$-South regions separately. The ${ }^{13} \mathrm{CO} 2 \rightarrow 1$ data are publicly available as

4 The Two Micron All Sky Survey (2MASS) is a joint project of the University of Massachusetts and the Infrared Processing and Analysis Center/California Institute of Technology, funded by the National Aeronautics and Space Administration and the National Science Foundation. a fits-data cube on www.ph1.uni-koeln.de/workgroups/ obs_astronomy/cygnusx.

\subsection{The CygX-North region}

\subsection{1. ${ }^{13} \mathrm{CO} 2 \rightarrow 1$ and $3 \rightarrow 2$ maps}

The ${ }^{13} \mathrm{CO} 2 \rightarrow 1$ emission distribution of the CygX-North region (northern part of the map displayed Fig. 2) shows many more structural details compared to the CfA survey $\left({ }^{12} \mathrm{CO} 1 \rightarrow 0\right.$ map at 8.7 angular resolution, Leung et al. 1992). This is not only due to the higher angular resolution, but also to the more optically thin ${ }^{13} \mathrm{CO}$ line used. While the CfA survey shows almost no holes in molecular emission and a rather smooth emission distribution towards the entire region, individual molecular clouds characterized by higher column density can clearly be identified in the KOSMA map. The ${ }^{13} \mathrm{CO} 3 \rightarrow 2$ map (Fig. A.1) allows even more pronounced emission peaks to be identified due to the higher density required for excitation of the $3 \rightarrow 2$ line (tend to be found in the denser cloud interiors).

The molecular clouds in the ${ }^{13} \mathrm{CO} 2 \rightarrow 1$ map are typically elongated filaments on a size-scale of around $10^{\prime}$ to $20^{\prime}$ (5 to $10 \mathrm{pc}$ at $1.7 \mathrm{kpc}$ distance). Smaller scale substructure and more diffuse emission is found between the large cloud fragments. The $\mathrm{H}$ II regions DR20 and DR21 and the young stellar object (YSO) W75N are embedded in molecular clouds, while DR17, DR18, DR22, and DR23 are located at the edges of or well-separated from molecular clouds. The typical main-beam brightness temperature of ${ }^{13} \mathrm{CO} 2 \rightarrow 1$ is around $5 \mathrm{~K}$ at the emission peaks and reaches up to $10 \mathrm{~K}$ in the DR21 region (see Table C.1). This emission is highly beam-diluted since additional ${ }^{13} \mathrm{CO} 2 \rightarrow 1$ observations with the IRAM $30 \mathrm{~m}$ telescope (Schneider et al. 2006, in prep.) show main-beam brightness temperatures of up to $30 \mathrm{~K}$ in the $\mathrm{DR} 21$ region.

The mere detection of the higher excited ${ }^{13} \mathrm{CO} 3 \rightarrow 2$ line in all regions emitting in ${ }^{13} \mathrm{CO} 2 \rightarrow 1$ indicates that the main cloud clumps are rather warm $(20-30 \mathrm{~K})$. They are thus most likely star formation sites and not quiescent, cold molecular cloud cores.

\subsubsection{Channel maps of ${ }^{13} \mathrm{CO} 2 \rightarrow 1$ emission}

The complexity of both the spatial and the velocity distribution of the molecular clouds in the ${ }^{13} \mathrm{CO}$ data requires a careful analysis of the different velocity channels. In order to identify coherent groups and their possible physical relation, we used channel maps of the ${ }^{13} \mathrm{CO} 2 \rightarrow 1$ intensity displayed in Fig. 3 .

(1) The DR22-DR23 filament: from $v=-8$ to $-2.8 \mathrm{~km} \mathrm{~s}^{-1}$, ${ }^{13} \mathrm{CO}$ emission is dominated by a north-south oriented, elongated molecular cloud connecting DR23 and DR22 and showing a velocity gradient of $\sim+6 \mathrm{~km} \mathrm{~s}^{-1}$. The complex 
100

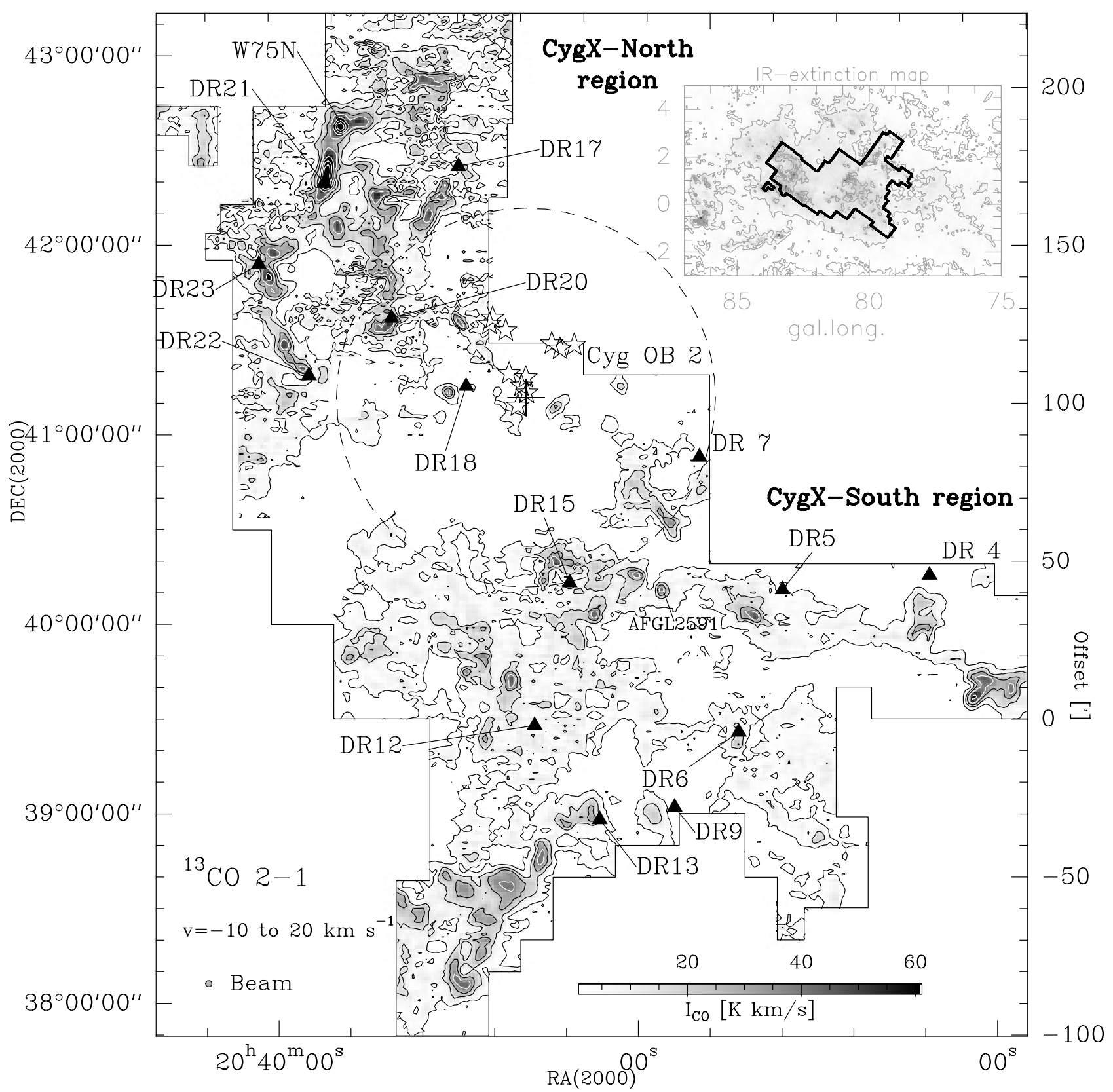

Fig. 2. Line integrated $\left(v=-10\right.$ to $\left.20 \mathrm{~km} \mathrm{~s}^{-1}\right){ }^{13} \mathrm{CO} 2 \rightarrow 1$ emission from Cygnus $\mathrm{X}$ as observed with KOSMA in grey scale. Contour lines are drawn from $9 \sigma$ to $45 \sigma$ in steps of $18 \sigma$ (black) and $63 \sigma$ to $153 \sigma$ in steps of $30 \sigma$ (white) with $3 \sigma=0.35 \mathrm{~K} \mathrm{~km} \mathrm{~s}^{-1}$. Black triangles mark the position of radio sources, stars the brightest members of Cygnus OB2, and the dashed circle the approximate extent of the OB 2 cluster (Uyaniker et al. 2001). In the upper right corner, we reproduce the extinction map of Fig. 1 in Galactic coordinates and indicate the ${ }^{13} \mathrm{CO} 2 \rightarrow 1$ mapping region.

splits into two separate clouds around $-4 \mathrm{~km} \mathrm{~s}^{-1}$, which are correlated with the DR22 and DR23 H II regions.

(2) The DR21 complex and DR20: from $v=-5.4$ to $1.1 \mathrm{~km} \mathrm{~s}^{-1}$, another north-south oriented, bright ${ }^{13} \mathrm{CO}$ ridge centered on DR21 becomes apparent. The smooth velocity-position transition (see channel $-5.4 \mathrm{~km} \mathrm{~s}^{-1}$ ) with a velocity gradient of $\sim+3 \mathrm{~km} \mathrm{~s}^{-1}$ from north-west of DR23 to north of DR21 suggests that the DR22-DR23 filament is connected to the DR21 complex. The northern cloud of the DR2 $1{ }^{13} \mathrm{CO}$ ridge at $v \sim-2.8 \mathrm{~km} \mathrm{~s}^{-1}$ is located just west of $\mathrm{W} 75 \mathrm{~N}$ (which is itself visible at $\left.+9 \mathrm{~km} \mathrm{~s}^{-1}\right)$. Three clouds in the west and southwest of DR21 seem to be part of the DR21 complex (smooth and connected velocity-position features, see channel
$-1.4 \mathrm{~km} \mathrm{~s}^{-1}$ ). The two clouds associated with DR20 (referred to in the following as the DR20 and DR20W clouds; $v=-4$ to $0 \mathrm{~km} \mathrm{~s}^{-1}$ ) have ${ }^{13} \mathrm{CO}$ emission at the same velocities as the DR21 complex and show the same progressive velocity shift from east $\left(v \sim-6 \mathrm{~km} \mathrm{~s}^{-1}\right)$ to west $(v \sim$ $-1 \mathrm{~km} \mathrm{~s}^{-1}$ ).

(3) Diffuse emission: from $v=\sim 1.0$ to $6.4 \mathrm{~km} \mathrm{~s}^{-1}$, the ${ }^{13} \mathrm{CO}$ emission is diffuse and widespread across the whole mapped region. Coherent complexes are found north of DR17 and in the map center at $(0,0)$.

(4) The W75N/AFGL2620 complex and the DR17 pillars: between $\sim+7.7$ and $\sim+14 \mathrm{~km} \mathrm{~s}^{-1},{ }^{13} \mathrm{CO}$ emission is dominated by the molecular cloud associated with W75N and an 


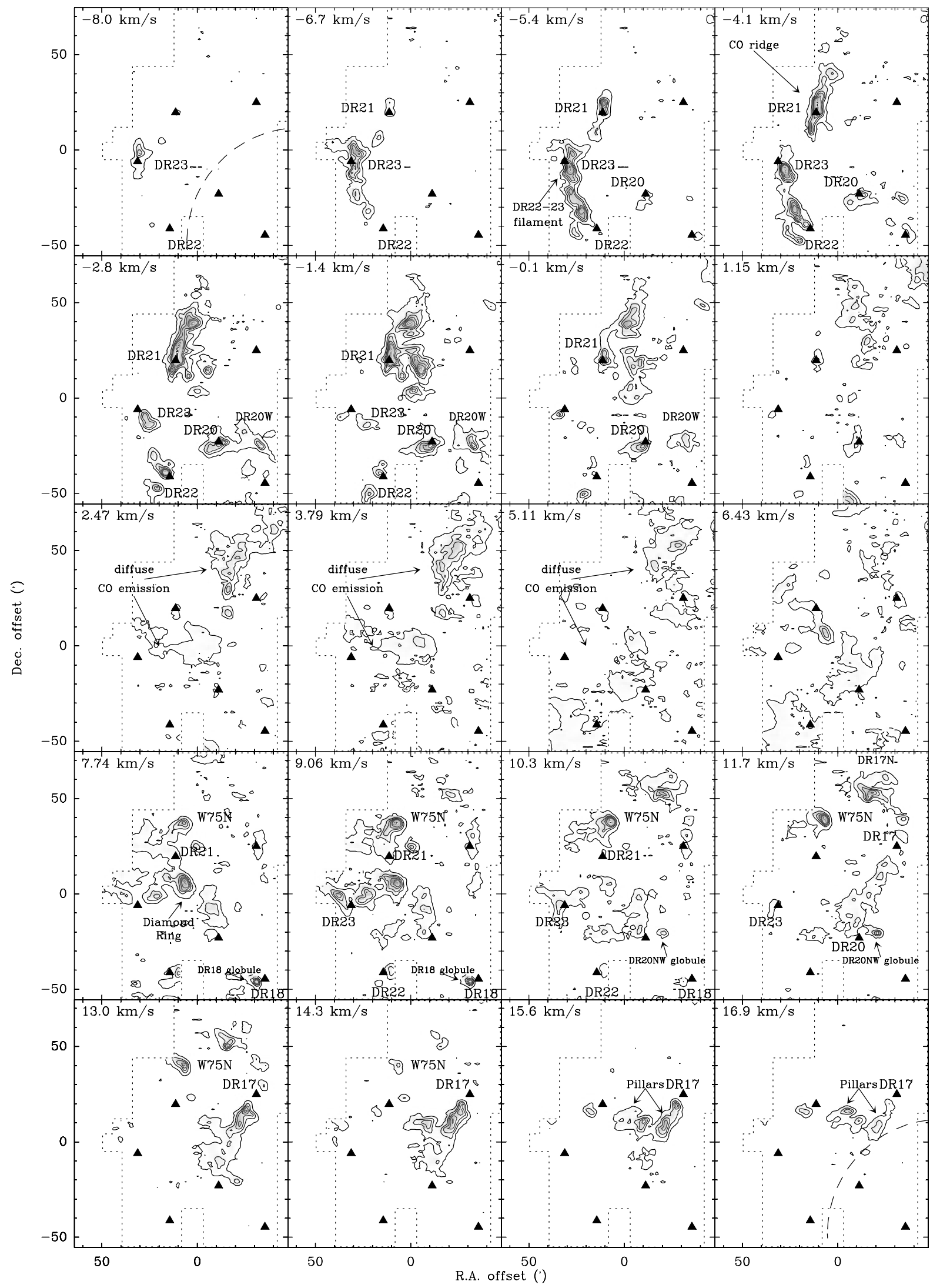

Fig. 3. Channel maps of ${ }^{13} \mathrm{CO} 2 \rightarrow 1$ emission with a step size of $\sim 1.3 \mathrm{~km} \mathrm{~s}^{-1}$ in the CygX-North region are shown. The lowest contour is the $6 \sigma$ level and contours go then in $6 \sigma$ steps. Triangles mark the positions of the H II regions DR17 to DR23. These sources are labeled only in the relevant velocity interval. Prominent features (filaments, globules, etc.) mentioned in the text are denoted by arrows. The dashed circle in the first and last channel shows the extent of the OB 2 association. 


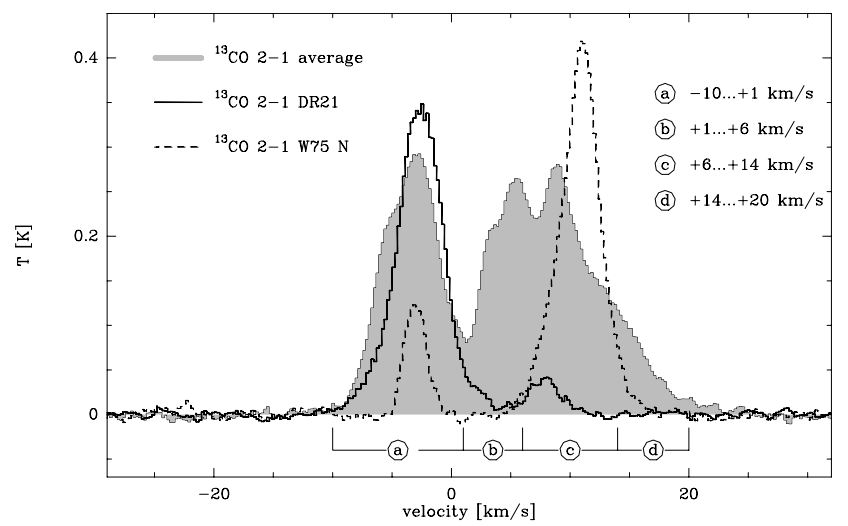

Fig. 4. The spatially-averaged spectrum of ${ }^{13} \mathrm{CO} 2 \rightarrow 1$ emission is displayed in grey. Single ${ }^{13} \mathrm{CO} 2 \rightarrow 1$ spectra taken from the DR21 and $\mathrm{W} 75 \mathrm{~N}$ regions (reduced by a factor of 30 and 20, respectively) are shown as a straight and dashed line. The main velocity ranges with ${ }^{13} \mathrm{CO}$ emission are indicated and labeled.

ensemble of clouds located at the center of the map between the AFGL2620 region (Marston et al. 2004, refer to this region as the "Diamond Ring" in their Spitzer mid-infrared images) and the position of DR23. Around $12 \mathrm{~km} \mathrm{~s}^{-1}$ an additional cloud (DR17N) emerges north of DR17 and northwest of W75N. From $v=+13$ to $+16.9 \mathrm{~km} \mathrm{~s}^{-1}$, three elongated, cometary-shaped clouds to the south and south-east of DR17 show up ("pillars"). All these clouds show a clear cometary shape pointing to what could be a still unknown strong source of UV radiation in the west. In contrast, the two clouds around DR23 do not show any sign of external influence from that source and are thus not necessarily part of the complex.

(5) The DR18 and DR20NW clouds: from $v=+7.7$ to $+10.3 \mathrm{~km} \mathrm{~s}^{-1}$ and +10.3 to $+13 \mathrm{~km} \mathrm{~s}^{-1}$, respectively, two small clouds associated with DR18 and with a region close to DR20 (referred to in the following as DR20NW) appears successively: the average velocity is $8.5 \mathrm{~km} \mathrm{~s}^{-1}$ for the DR18 cloud and $11.5 \mathrm{~km} \mathrm{~s}^{-1}$ for DR20NW. Both clouds have a globular shape and clearly point to the center of Cyg OB2. They are shaped by the radiation and stellar winds of the cluster members.

\subsubsection{Spatially averaged ${ }^{13} \mathrm{CO}$ spectra}

The positional averaged spectrum across the part of the ${ }^{13} \mathrm{CO} J=$ $2 \rightarrow 1$ map characterized by the CygX-North region, i.e., all spectra north of approximately Dec $=40^{\circ} 30^{\prime}$, is displayed in Fig. 4, together with single spectra taken at the peak positions of DR21 $\left(-3 \mathrm{~km} \mathrm{~s}^{-1}\right)$ and W75N $\left(+9 \mathrm{~km} \mathrm{~s}^{-1}\right)$. The average spectrum reveals several, partially overlapping, line components across a very broad range of velocities $\left(-10\right.$ to $\left.20 \mathrm{~km} \mathrm{~s}^{-1}\right)$. See Sect. 6.4 for a discussion of the large velocity coverage.

Nevertheless, we emphasize that, since radial velocities cannot easily be converted into a distance in Cygnus X, this large spread of observed velocities around the tangent point velocity $\left(3-4 \mathrm{~km} \mathrm{~s}^{-1}\right)$ does not imply that we observe molecular clouds stretched along the line of sight between 1 and typically a few kpc. Based on the channel maps and the averaged spectrum, four main velocity ranges can be defined: (a) from -10 to $1 \mathrm{~km} \mathrm{~s}^{-1}$; (b) from 1 to $6 \mathrm{~km} \mathrm{~s}^{-1}$; (c) from 6 to $14 \mathrm{~km} \mathrm{~s}^{-1}$, and (d) from 14 to $20 \mathrm{~km} \mathrm{~s}^{-1}$. They roughly represent the DR20-2122-23 complex, the diffuse gas, the W75N-AFGL2620 clouds, and the DR17 pillars, respectively. Although this definition is somewhat arbitrary, since the lines are not well-separated, we use these ranges in the following to characterize the different emission regions (see Figs. 7 to 9).

\subsection{The CygX-South region}

\subsection{1. ${ }^{13} \mathrm{CO} 2 \rightarrow 1$ and ${ }^{12} \mathrm{CO} 3 \rightarrow 2$ maps}

CygX-South constitutes the southern part $\left(\mathrm{Dec}<40^{\circ} 30^{\prime}\right)$ of the ${ }^{13} \mathrm{CO} 2 \rightarrow 1$ emission distribution seen in the velocity integrated map of Fig. 2. It includes all molecular clouds associated with the sources DR4-7, 9, 12, 13, and 15. A part of this area was observed in the ${ }^{12} \mathrm{CO} 3 \rightarrow 2$ line as shown in Fig. A.2.

Compared to $\mathrm{CygX}$-North, both the ${ }^{13} \mathrm{CO} 2 \rightarrow 1$ and the ${ }^{12} \mathrm{CO} 3 \rightarrow 2$ emission appear generally more diffuse with less clearly defined clouds and cloud fragments. A prominent ${ }^{13} \mathrm{CO} 2 \rightarrow 1$ emission feature extends from east of DR15 to the south-west of DR4 and is associated with the dark cloud L889, which crosses in front of and apparently bisects the bright $\mathrm{H}$ II region IC $1318 \mathrm{~b} / \mathrm{c}$ seen in the optical (Fig. 1). While the dark cloud appears as a continuous band of emission in the CfA ${ }^{12} \mathrm{CO} 1 \rightarrow 0$ map (Fig. 1), tracing lower density material, it is more fragmented in ${ }^{13} \mathrm{CO} 2 \rightarrow 1$ with regions of peak emission, and thus higher (column) density, directly linked to the $\mathrm{H}$ II regions DR5 and DR15. A region of high ${ }^{13} \mathrm{CO}$ and ${ }^{12} \mathrm{CO}$ intensity is found southeast of DR13 (marked "DR13S") where several distinct emission peaks characterize the cloud structure. None of these clouds is associated with a radio continuum source. The ${ }^{13} \mathrm{CO}$ emission close to DR7 is not physically associated with the DR7 $\mathrm{H}$ II region since the latter is actually located in the Perseus arm at $\mathrm{CO}$ velocities around $-50 \mathrm{~km} \mathrm{~s}^{-1}$, which are not part of the velocity integrated map shown in Fig. 2.

The typical main-beam brightness temperatures of ${ }^{13} \mathrm{CO} 2 \rightarrow 1$ in the southern part of Cygnus $\mathrm{X}$ are lower than in the CygX-North region, up to $8 \mathrm{~K}$ at the emission peaks of DR4/DR15 and a few $\mathrm{K}$ in the lower intensity regions. The ${ }^{13} \mathrm{CO} 3 \rightarrow 2$ emission (map not shown due to the poor and irregular coverage) was detected towards the molecular peaks at temperatures of typically a few $\mathrm{K}\left({ }^{12} \mathrm{CO} 3 \rightarrow 2\right.$ main-beam brightness temperature around $10-20 \mathrm{~K}$, see Table C.2).

\subsubsection{Channel maps of ${ }^{13} \mathrm{CO} 2 \rightarrow 1$ emission}

Channel maps of the ${ }^{13} \mathrm{CO} 2 \rightarrow 1$ emission in the CygX-South region are displayed in Fig. 5. The degree of confusion for the emission from individual clouds and cores is higher than for the CygX-North region, making it difficult to separate individual features. Still, a number of coherent cloud complexes can be identified.

(1) The OB2 globules and the AFGL2591 cloud: in the north of the map, two compact clouds with bulk emission at $v \sim-10$ and $-4.5 \mathrm{~km} \mathrm{~s}^{-1}$ are located in projection close to the center of the Cyg OB2 association. Their proximity to the cluster and high degree of compactness is a strong indication of being influenced and shaped by the Cyg OB2 UV radiation and stellar winds. At the center of the map, at $v \sim$ $-6 \mathrm{~km} \mathrm{~s}^{-1}$, the parent cloud of the well-known massive protostar AFGL2591 is recognized as a compact clump with a cometary shape pointing to the center of Cyg OB2. Weaker, more diffuse emission in the west most probably represents a physically associated cloud. 


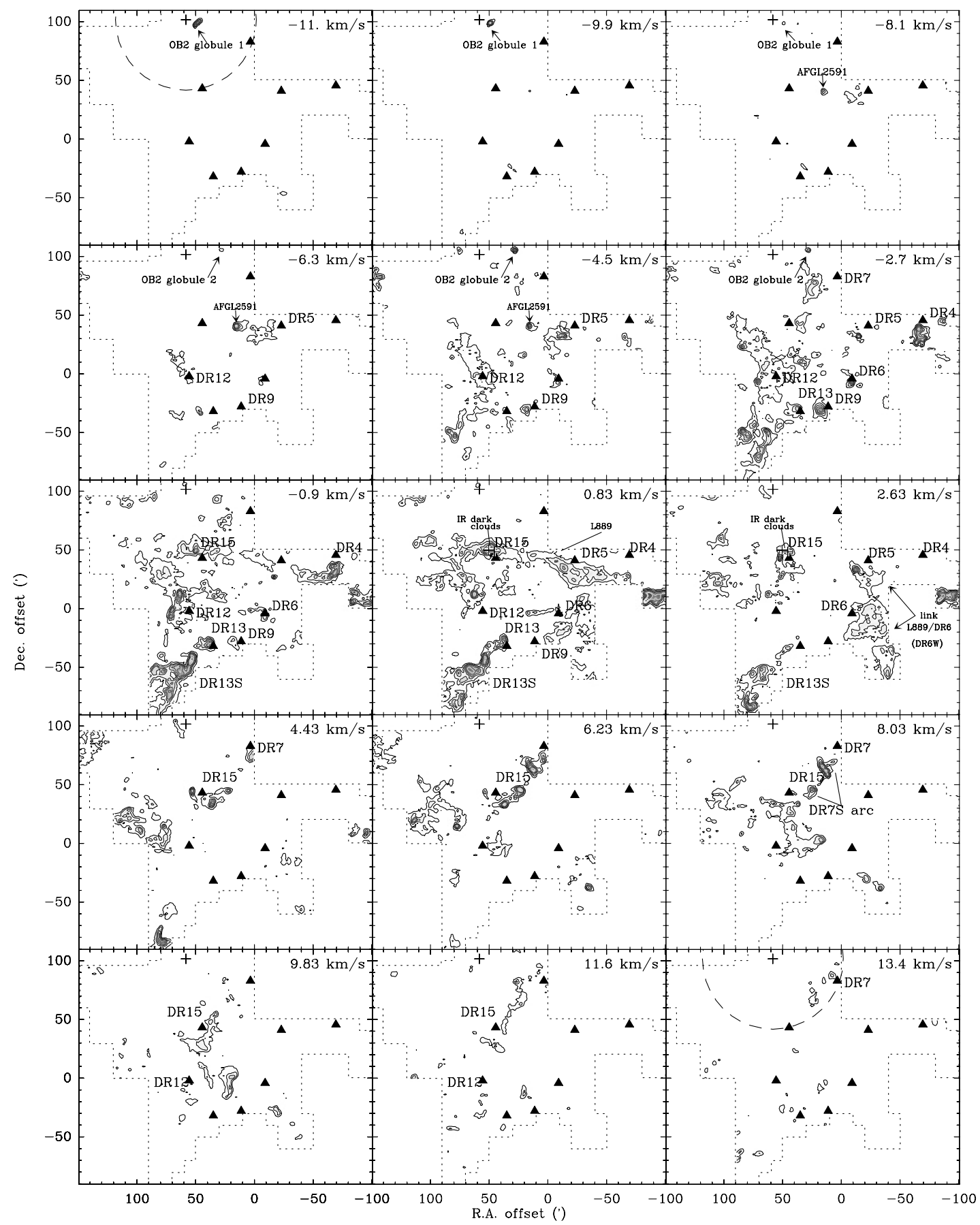

Fig. 5. Channel maps of ${ }^{13} \mathrm{CO} 2 \rightarrow 1$ emission with a step size of $\sim 1.4 \mathrm{~km} \mathrm{~s}^{-1}$ in the CygX-South region are shown. The lowest contour is the $9 \sigma$ level and contours go then in $6 \sigma$ steps. Triangles mark the positions of the H II regions DR4 to DR 15 . These sources are labeled only in the relevant velocity interval. The cross indicates the center of the OB 2 cluster. The dashed circle in the first and last channel shows the extent of the OB 2 association. Prominent features mentioned in the text are denoted by arrows.

(2) The DR4-DR5-L889 dark lane complex: at $v=-2.7 \mathrm{~km} \mathrm{~s}^{-1} \mathrm{a}$ compact and clumpy cloud is visible just south of the supernova remnant DR4. Towards more positive velocities (best visible in channel $\left.-0.9 \mathrm{~km} \mathrm{~s}^{-1}\right)$, this cloud extends to the east, following the dark lane L889 (see Fig. 1), and reaches across DR15 (at $v=0.83 \mathrm{~km} \mathrm{~s}^{-1}$ ). In the far west of the map, another compact, high-intensity cloud shows up, which is probably part of the same DR4/L889 complex.

(3) The DR6 and DR6W clouds: the cloud associated with DR6 is visible for $v=-2.7$ to $-0.9 \mathrm{~km} \mathrm{~s}^{-1}$. It is compact and has the same CO velocity as the DR4-L889-DR15 group, but does not show any other indication that it could be physically associated with that complex. Slightly west of DR6 a more diffuse and larger emission region at $v \sim+2.6 \mathrm{~km} \mathrm{~s}^{-1}$ defines a CO cloud (referred to in the following as the DR6W cloud), which does show indications of being associated with L889 (see the smooth velocity-position transition from $v=+0.8$ to $+2.6 \mathrm{~km} \mathrm{~s}^{-1}$ and the emission bridge at $v=+2.6 \mathrm{~km} \mathrm{~s}^{-1}$ ). It could be part of a ring structure also comprising DR15, DR12, and L889 (see below).

(4) The DR12-DR15 ring complex: from $v=-2.7$ to $0.83 \mathrm{~km} \mathrm{~s}^{-1}$, a diffuse cloud in the north of DR12 evolves 


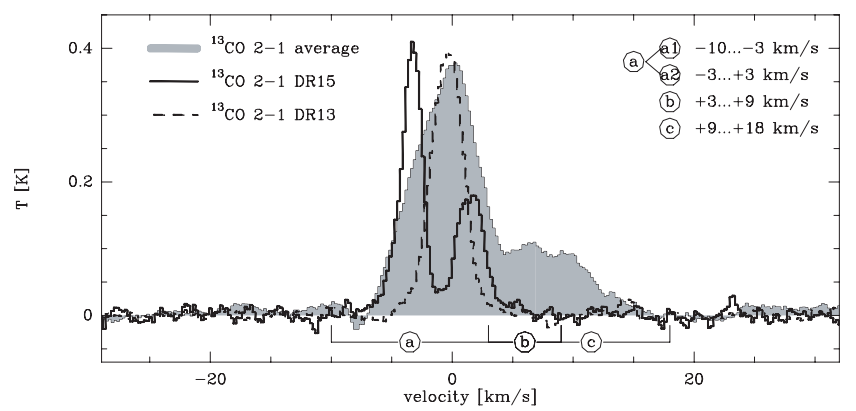

Fig. 6. Spatially-averaged spectrum of ${ }^{13} \mathrm{CO} 2 \rightarrow 1$ emission (grey) together with single ${ }^{13} \mathrm{CO} 2 \rightarrow 1$ spectra taken from the DR15 and DR13 regions (straight and dashed line, both reduced by a factor of 15 ). The main velocity ranges with ${ }^{13} \mathrm{CO}$ emission are indicated and labeled.

in shape from an arc pointing to the DR22-DR23 regions (north-east) and to the DR13 region (south), to a portion of a ring that encompasses the DR15 and DR6W clouds and possibly L889. Several compact clouds are superposed on top of this plateau of diffuse emission. A narrow filament extends from DR15 to the east of DR12 (channel -2.7 to $-0.9 \mathrm{~km} \mathrm{~s}^{-1}$ ) where two compact, elongated clumps show up. For $v=-0.83$ to $+0.9 \mathrm{~km} \mathrm{~s}^{-1}$, the bulk of the DR 15 cloud appears as a ring of emission that connects to the DR12 cloud and L889. Close to the nominal position of DR15, two infrared dark clouds (IRDCs, Egan et al. 1998; Oka et al. 2001) can be identified. They both have the same velocity range of $\sim-1.5$ to $\sim 2.5 \mathrm{~km} \mathrm{~s}^{-1}$.

(5) The DR13 filament and the DR9 cloud: between $v=\sim-2.7$ and $+2.6 \mathrm{~km} \mathrm{~s}^{-1}$, a chain of clouds extends from DR13 to the south east. The smooth velocity-position transition $(v=-5$ to $-2 \mathrm{~km} \mathrm{~s}^{-1}$ ) from the DR12 diffuse cloud to the south eastern part of the DR13 cloud indicates that these two complexes are associated. The DR13 cloud consists of a filament of compact clouds close to DR13 and splits into a more diffuse V-shaped cloud in the south east. This morphology may have been shaped by winds or radiation coming from an embedded, unknown source close to DR13 or from either the Cyg OB2 or OB9 association, although the latter are rather far away from the DR13 cloud.

A compact cloud at $v=-4.5$ to $-0.9 \mathrm{~km} \mathrm{~s}^{-1}$ is associated with DR9. This cloud and the DR13 cloud can actually be part of the same complex, since a smooth velocity-position transition in the channels from -4.5 to $-0.9 \mathrm{~km} \mathrm{~s}^{-1}$ is visible. DR9 could be the cloud of the DR13 complex, which is the closest, and thus the most exposed, to the Cyg OB2 and/or OB9 associations making it compact and not connected to DR13 anymore. Finally, we note that the far south side of the DR13 cloud also seems to be externally shaped/irradiated. The source of this UV radiation, which would be located in the south, is unknown.

(6) The DR7S arc complex: at velocities between +4.4 and $+11.6 \mathrm{~km} \mathrm{~s}^{-1}$, a coherent group of clouds is visible from the south of DR7 to the east of DR12-DR15. These clouds are arranged along a large-scale arc centered on OB2 with compact clouds in the north (between DR7 and DR15) and more diffuse clouds in the south (east of DR12-DR15).

\subsubsection{Spatially averaged CO spectra}

Figure 6 displays the average spectrum for the whole CygXSouth region and individual spectra of ${ }^{13} \mathrm{CO} 2 \rightarrow 1$ line emission taken at peak positions of the molecular clouds associated with DR13 and DR15. Although the profile of the averaged ${ }^{13} \mathrm{CO}$ line appears less complex than in the CygX-North region, it is actually still composed of a number of individual components, partly overlapping in velocity. This is evident from the ${ }^{13} \mathrm{CO}$ lines in the DR13 and DR15 regions, which have quite different velocities towards the emission peaks. The large velocity breadth of $\mathrm{CO}$ emission will be discussed in Sect. 6.4. Considering the other molecular cloud complexes, we arrive at the following velocity intervals best describing the main features visible in the data for CygX-South similar to the ranges defined for the DR21 region: (a1) -10 to $-3 \mathrm{~km} \mathrm{~s}^{-1}$, (a2) -3 to $3 \mathrm{~km} \mathrm{~s}^{-1}$ (for simplicity sometimes comprised into range (a) -10 to $3 \mathrm{~km} \mathrm{~s}^{-1}$ ), (b) 3 to $9 \mathrm{~km} \mathrm{~s}^{-1}$, (c) 9 to $18 \mathrm{~km} \mathrm{~s}^{-1}$.

\section{Mid-infrared emission from MSX: probing the association of molecular clouds with young OB stars and embedded IR sources}

The central part of the Cygnus $\mathrm{X}$ region, as marked in Fig. 1, is shown in more detail in ${ }^{13} \mathrm{CO} 2 \rightarrow 1$ emission (contours) over 3 velocity intervals (Figs. 7 to 9), overlaid on a grey(color) scale image of mid-IR emission as obtained with MSX (Band A at $8.3 \mu \mathrm{m}$ with an angular resolution of $\left.20^{\prime \prime}\right)$. The velocity intervals for the integration in the CygX-North and CygX-South regions have been defined in order to best reproduce the $\mathrm{CO}$ distribution of the coherent complexes as defined in Sects. 4.1.3 and 4.2.3 with as few integration ranges as possible.

The extended emission at $8 \mu \mathrm{m}$ mainly traces UV heated small grains and polycyclic aromatic hydrocarbons (PAHs) in photon dominated regions (PDRs). Sources of the UV radiation can be early B (B0-B1) stars. The MSX image thus emphasizes interfaces between molecular clouds and intense UV fields from young massive stars. It also contains point-like sources that are either young massive and bright stars or embedded red objects, which are usually massive protostars. In the following, we review links between groups of clouds identified in the $\mathrm{CO}$ maps and signs of present and recent star formation activity, such as OB associations, infrared clusters, and bright IRAS/MSX sources in order to recognize large coherent molecular complexes and to ultimately describe the 3D distribution of objects towards Cygnus X. The location of clusters and individual IR-sources (identified in the IRAS and MSX-catalogs) is given in detail in Figs. B. 1 to B.6, which show ${ }^{13} \mathrm{CO} 2 \rightarrow 1$ maps in selected velocity ranges for each region in order to define molecular clumps. These IR sources are selected from the MSX point source catalog ${ }^{5}$ as sources with a flux at $21 \mu \mathrm{m}$ significantly larger than at $8 \mu \mathrm{m}$ in terms of $F_{v}$ (see Bontemps et al. 2006 , in prep.). These sources are referred to in the following as MSX "red" sources.

\subsection{The DR20, DR21, DR22, and DR23 regions: $v_{\mathrm{CO}}$ from -10 to $+1 \mathrm{~km} \mathrm{~s}^{-1}$}

At $v_{\mathrm{CO}}$ from -10 to $+1 \mathrm{~km} \mathrm{~s}^{-1}$ (Fig. 7), the CygX-North region consists of clearly-defined CO-clumps with strong emission gradients. There is often a close association of MSX emission with CO clumps (rim brightening of mid-IR emission at the edges of the CO clumps), indicating that there is indeed a physical association between the $\mathrm{OB}$ stars and the molecular clouds.

The DR22-DR23 CO filament is associated with a network of filaments prominent in mid-IR emission tracing PDR

\footnotetext{
${ }^{5}$ Version 2.3 of the Midcourse Space Experiment (MSX).
} 


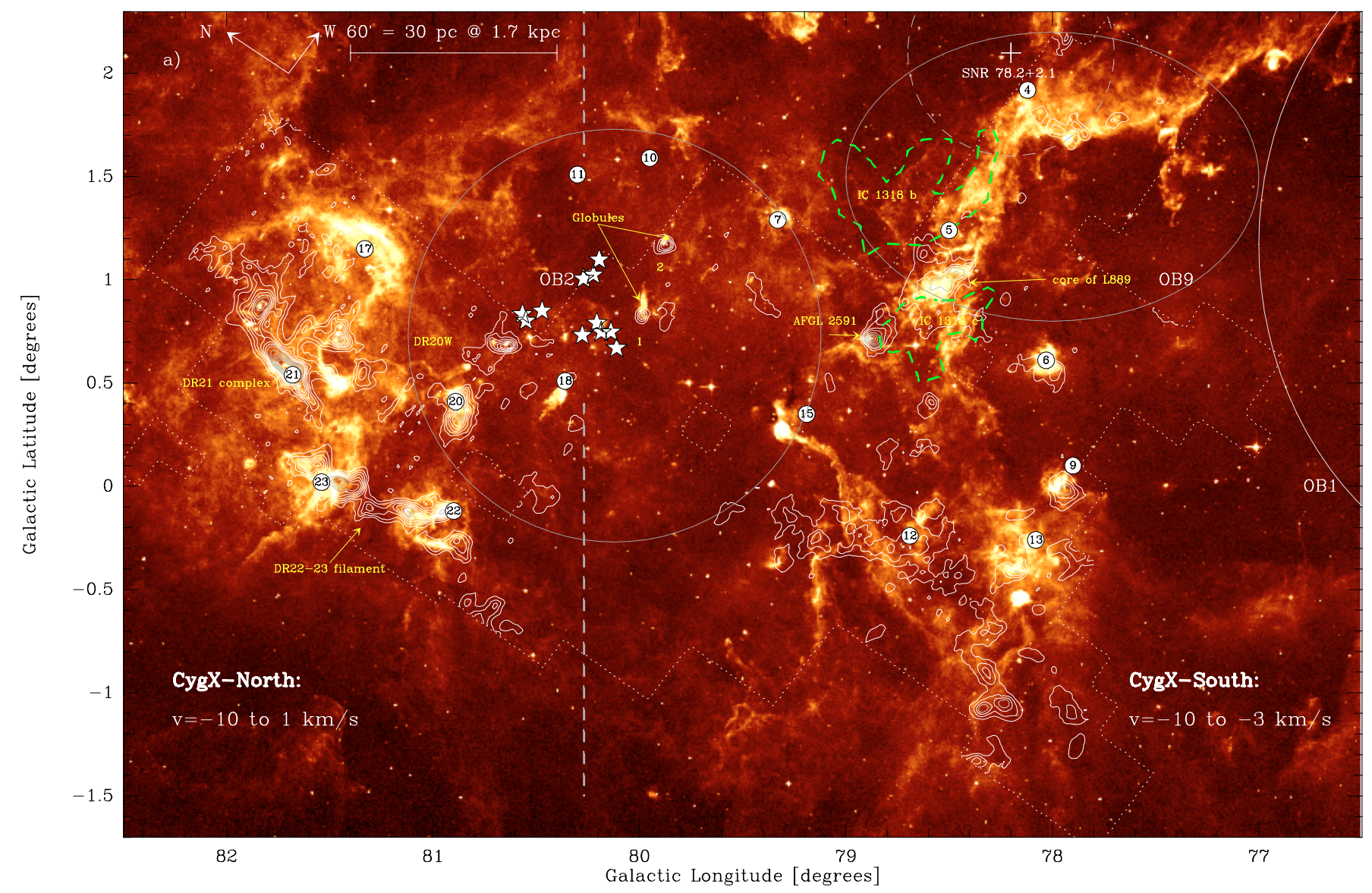

Fig. 7. Overlay of ${ }^{13} \mathrm{CO} 2 \rightarrow 1$ emission (contours) on $8 \mu \mathrm{m}$ emission (MSX). The grey(color) scale goes from 0 to $1.2 \times 10^{-5} \mathrm{~W} \mathrm{~m} \mathrm{~m}^{2} \mathrm{sr}$. The velocity ranges for the CygX-North region and CygX-South region are slightly different $\left(-10\right.$ to $1 \mathrm{~km} \mathrm{~s}^{-1}$ and -10 to $\left.-3 \mathrm{~km} \mathrm{~s}^{-1}\right)$. A vertical dashed line indicates the border between the two regions. Contours go from 2.5 to $15 \mathrm{~K} \mathrm{~km} \mathrm{~s}^{-1}$ in steps of $2.5 \mathrm{~K} \mathrm{~km} \mathrm{~s}^{-1}$ (indicated as $2.5 / 15 / 2.5 \mathrm{~K} \mathrm{~km} \mathrm{~s}$ from now on) and 17.5/42.5/5 K km s${ }^{-1}$ for the CygX-North region and 1.5/4.5/1.5 K km s${ }^{-1}$ and 6/24/3 for the CygX-South region. The small-dotted polygon marks the extent of KOSMA mapping. The grey ellipses characterize the extent of the OB clusters OB 2 and 9 , and the stars give the positions of the most massive members of OB 2. Small circles with numbers indicate the H II regions DR4 to DR23. The long-dashed polygon outlines IC $1318 \mathrm{~b} / \mathrm{c}$ and the circle the SNR 78.2+2.1 and the cross its center.

interfaces due to early type stars close to the cloud. For DR23, these suspected OB stars are probably part of the weak infrared cluster [LK2002]Cl-15 (see Fig. B.3) recognized from 2MASS images by Le Duigou \& Knödlseder (2002), which is identical with the cluster identification [DB2001]Cl-17/18 by Dutra \& Bica (2001). The DR22 cloud contains the rich infrared cluster [LK2002]Cl-11 or [DB2001]Cl-14 (Fig. B.3) that is responsible for most of the PDR interfaces seen as extended emission in the MSX image. Two MSX point-like sources are embedded within the CO clumps of the DR22-DR23 filament whereof MSX6G80.9383-0.1268 (IRAS 20375+4109) is one of the brightest red mid-IR source in Cygnus X. It is most probably a mid-IR bright massive protostar. MSX6G81.1225-0.1343 is weaker and may correspond to an intermediate-mass protostar.

The DR21 complex consists of a molecular ridge (Fig. B.3) with several $\mathrm{CO}$ clouds that have been intensively studied in the past (e.g. Wilson \& Mauersberger 1990). A network of bright mid-IR filaments clearly coincides with $\mathrm{CO}$ emission and seems to originate inside the ridge. Mid-IR emission is particulary bright toward the DR21 object itself and in the eastern part of the $\mathrm{CO}$ ridge. The $\mathrm{CO}$ ridge coincides with an IR-dark filament seen with MSX (see also the Spitzer image in Marston et al. 2004), which suggests that at least some of the CO clouds at $-3 \mathrm{~km} \mathrm{~s}^{-1}$ are in front of the sources of UV radiation. No embedded cluster has been recognized so far in the DR21 ridge, suggesting that the UV radiation sources are deeply embedded and/or too young objects to show up as well-developed OB clusters (see Bontemps et al. 2006, in prep. for a detailed discussion of the nature of infrared sources in Cygnus X). DR21 is an UCH II region associated with a powerful outflow and coinciding with MSX6G81.6802+0.5405, which might well be an early stage OB cluster. Three arcmin north of DR21, a group of three infrared sources constitutes the DR21 $(\mathrm{OH})$ region. The weak source MSX6G81.7220+0.5699 coincides with DR21(OH) itself, which is a double millimeter source with 7" separation (Mangum et al. 1991) and a candidate for a massive protostar in an early stage. West of $\mathrm{DR} 21(\mathrm{OH}), \mathrm{MSX6G81.7131+0.5792}$ is a red MSX source that coincides with a single 2 MASS source and could be a young early-type star with large infrared excess. It does not coincide with the millimeter source DR21(OH)-W from Richardson et al. (1989). South of DR21(OH), MSX6G81.7133+0.5589 is also a red mid-IR source, but it is not identical to the nearby source DR21(OH)-S (Richardson et al. 1989).

The mid-IR emission correlated with the north, west, and south-west CO clumps is generally much weaker than toward the main ridge. When present, mid-IR emission is spatially confined and mainly coincides with point-like MSX sources. On the other hand, four MSX sources in the region, including DR21, W75SFIR3, and MSX6G81.7131+0.5792 (see also [DB2001]Cl-19), 


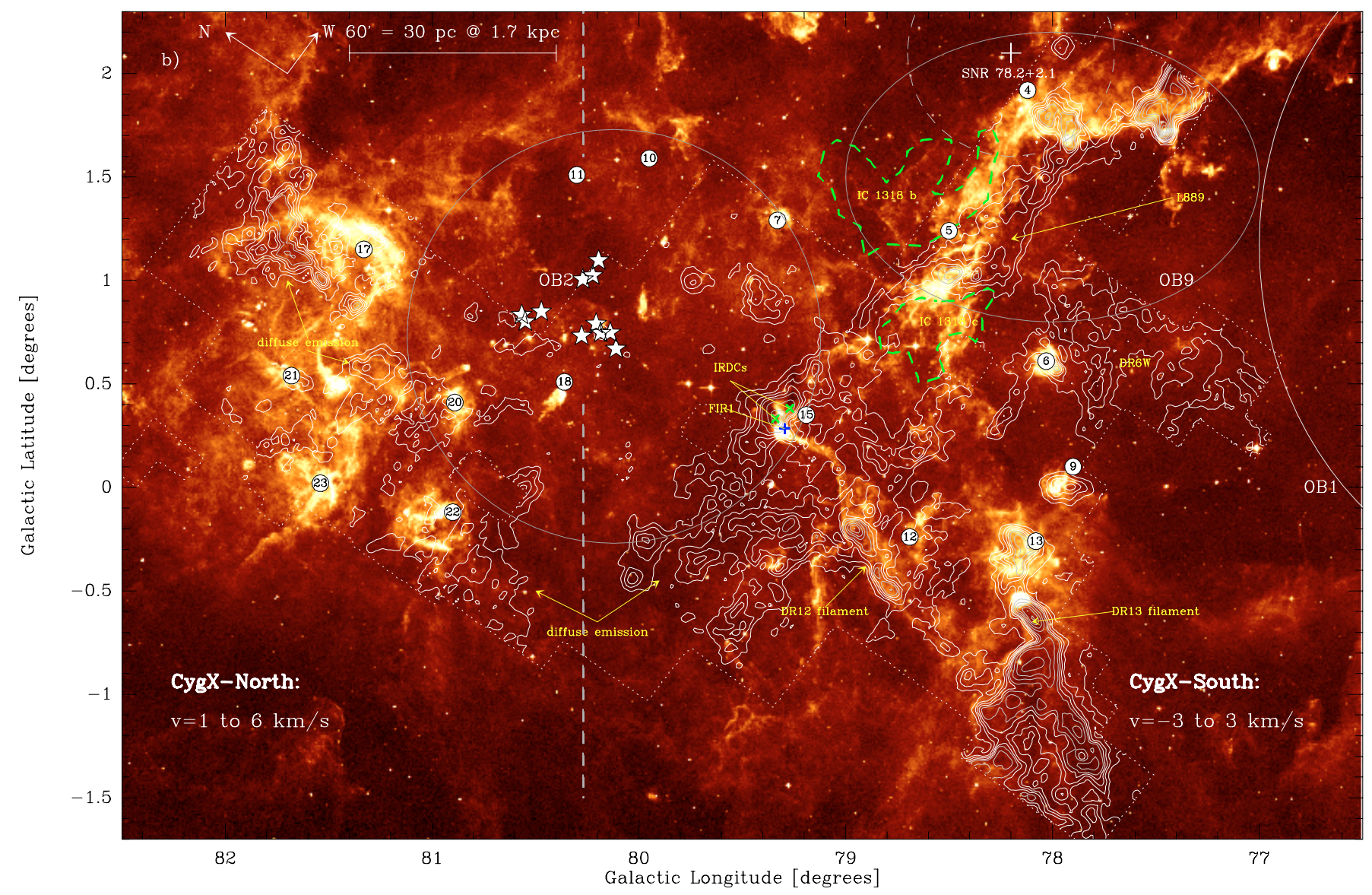

Fig. 8. The same as Fig. 7, but in the velocity range 1 to $6 \mathrm{~km} \mathrm{~s}^{-1}$ (CygX-North) and -3 to $3 \mathrm{~km} \mathrm{~s}^{-1}$ (CygX-South). Contours: $1.5 / 6 / 1.5 \mathrm{~K} \mathrm{~km} \mathrm{~s}{ }^{-1}$ and 7.5/37.5/3 K km s${ }^{-1}$ for CygX-North and $1.5 / 6 / 1.5 \mathrm{~K} \mathrm{~km} \mathrm{~s}^{-1}$ and 7.5/25.5/3 K km s${ }^{-1}$ for CygX-South. The white (blue) cross indicates the position of FIR1 (Odenwald et al. 1990), and the white (green) crosses the location of two Infrared Dark Clouds (Egan et al. 1998).

seem to be associated in 2MASS images with small groups of a few bright stars that could represent the seeds for the formation of larger clusters in the near future.

The DR20 clouds DR20-CO1 and DR20-CO2/DR20W (Fig. B.2) are associated with strong mid-IR emission. The two CO clumps both host an active star-forming region associated with strong IR sources that are good candidates to be bright infrared massive protostars. One of them was identified as a single O5-6 ZAMS star (Odenwald et al. 1990).

\subsection{The Cyg OB2 globules and the AFGL2591 cloud: $v_{\mathrm{CO}}$ from -10 to $-3 \mathrm{~km} \mathrm{~s}^{-1}$}

Two compact clouds (marked "globules" in Fig. 7) are associated with extended mid-infrared emission which is a signature of PDR interfaces due to OB2. Globule 2 is associated with two mid-IR sources.

The clump AFGL2591-CO1 (Fig. B.5) hosts one of the brightest infrared sources of Cygnus X, AFGL2591, a wellknown massive protostar (e.g., van der Tak et al. 1999 and references therein). Its cometary shape might be due to the compression from the ionization front of the OB 2 cluster (despite its rather large projected distance of $\sim 30 \mathrm{pc}$ to the OB2 cluster center, see Sect. 6.1). Some weak, rim-like, extended emission at the compressed edge of the cloud can be seen in the $8 \mu \mathrm{m}$ MSX image supporting the PDR interface interpretation.

\subsection{The DR4-DR5-L889, DR6, DR6W, DR9, and DR12-DR15 regions: $v_{\mathrm{CO}}$ from -3 to $+3 \mathrm{~km} \mathrm{~s}^{-1}$}

This velocity range (Fig. 8) contains the spatially most widespread ${ }^{13} \mathrm{CO}$ emission in Cygnus $\mathrm{X}$. A comparison with the optical image (Fig. 1) shows that the diffuse emission arises from a region of high visual extinction, i.e., the coherent DR4-DR5-L889 elongated CO complex that corresponds to the dark lane passing in front of the IC $1318 \mathrm{~b} / \mathrm{c}$ nebula. It has long been a subject of discussion (i) whether the $\mathrm{H}_{\text {II }}$ regions IC $1318 \mathrm{~b}$ and c are connected and (ii) whether the dust lane $\mathrm{L} 889$ is physically associated with the $\mathrm{H}$ II region(s) (Goudis \& Meaburn 1974; Dickel et al. 1977; Baars \& Wendker 1981; Campbell et al. 1981; Wendker et al. 1983). Our data show that the main $\mathrm{CO}$ emission peak close to DR5 is directly associated with large-scale, extended mid-IR emission. This correlation also becomes evident in maps of FIR $90 \mu \mathrm{m}$ emission shown in Campbell et al. (1981). Further west, towards DR4, mid-IR emission and peak CO emission are slightly displaced, which could be due to the overall geometry of the region. The excitation source could either be a single $\mathrm{O}$ star, as suggested by Ackermann et al. (1970), or a still unidentified population of OB stars lying inside IC $1318 \mathrm{~b} / \mathrm{c}$, or in the Cygnus OB9 association. The last, however, is assumed to be at a distance of $1.2 \mathrm{kpc}$ (Alter et al. 1970), which is slightly closer than the distance of $1.5 \mathrm{kpc}$ for IC $1318 \mathrm{~b} / \mathrm{c}$ (Dickel et al. 1969). Considering the large uncertainties in the distance estimates for both OB9 and IC $1318 \mathrm{~b} / \mathrm{c}$, it is likely that at the CO emission peak close to DR5, the $\mathrm{H}$ II region and the molecular gas, i.e., IC $1318 \mathrm{~b} / \mathrm{c}$ 


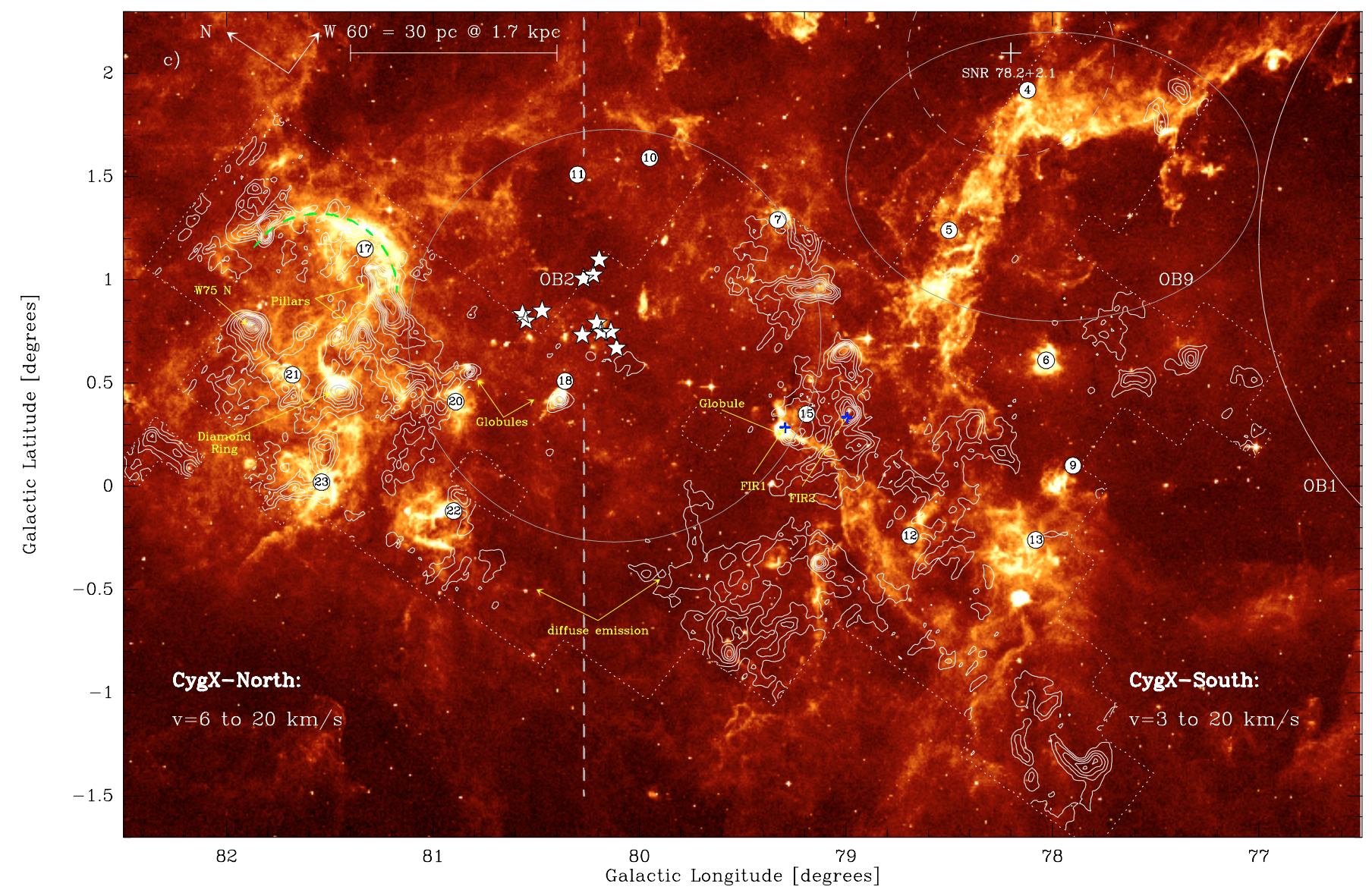

Fig. 9. The same as Fig. 7, but in the velocity range 6 to $20 \mathrm{~K} \mathrm{~km} \mathrm{~s}^{-1}$ (CygX-North) and 3 to $20 \mathrm{~K} \mathrm{~km} \mathrm{~s}^{-1}$ (IC $13818 \mathrm{~b} / \mathrm{c}$ ). Contours: 2.5/12.5/2.5 K km s${ }^{-1}$ and 15/40/5 K km s${ }^{-1}$ for CygX-North and 2/10/2 K km s${ }^{-1}$ and 12/24/4 $\mathrm{K} \mathrm{km} \mathrm{s}^{-1}$ for CygX-South. White (blue) crosses indicate the position of FIR1 and FIR2 (Odenwald et al. 1990). The black (green) dashed half-circle close to DR17N indicates the DR17 loop.

and L889, are in direct contact, whereas towards DR4, a slab of molecular gas is tilted away from the $\mathrm{H}_{\text {II }}$ region. Molecular emission at lower velocities $\left(-10\right.$ to $\left.-3 \mathrm{~km} \mathrm{~s}^{-1}\right)$ is also found at the mid-IR emission peak close to DR5. It is not clear whether this gas represents a molecular cloud placed behind or in front of the $\mathrm{H}$ II region. In all cases, we agree with the majority of views considering IC $1318 \mathrm{~b} / \mathrm{c}$ as a single, coherent $\mathrm{H}$ II region directly associated with L889.

We do not have any direct evidence that the supernova remnant G78.2+2.1 has an impact on the molecular cloud seen south of DR4 or on any other material in the region (as suggested by Landecker et al. 1980; and Wendker et al. 1991). For example, no shell structure in the molecular gas - also not in the ${ }^{13} \mathrm{CO}$ channel maps - is seen.

The east-west elongated DR15 CO complex (Fig. B.6) seems to be aligned along the main direction of the DR4-DR5-L889 dark lane cloud and could therefore be part of a coherent complex. The main DR15 CO clump (DR15-CO1, $15000 M_{\odot}$ ) has been a location of active star formation. It hosts the rich embedded cluster [LK2002]Cl-08 ([DB2001]Cl-10), which was already recognized by Odenwald et al. (1990) and Comeron \& Torra (2001). The main IR source IRAS 20306+4005 (FIR1 in Odenwald et al. 1990) sits at the tip of a column of weaker midIR emission pointing away from OB2. The associated molecular clump (DR15-CO4, Fig. B.6) follows the mid-IR emission exactly, and its bright rimmed morphology demonstrates the influence of the OB2 cluster. This becomes even more obvious at slightly higher velocities ( $>3 \mathrm{~km} \mathrm{~s}^{-1}$, see Sect. 5.6). These findings contradict the assumption of Cong (1977) that the molecular clouds of DR15 are foreground objects at a distance of around $1 \mathrm{kpc}$ and that they are unrelated to OB2. The two peaks of ${ }^{13} \mathrm{CO}$ emission found on either side of the FIR sources correspond to the IR dark clouds G79.27+0.38 and G79.34+0.33. Since they are coincident with visual obscuration, they must be located in front of the $\mathrm{H}_{\mathrm{HI}}$ region (Oka et al. 2001).

The emission from DR12 in this velocity range seems to form a ring structure, together with the DR 15 clumps. The two elongated clumps (DR12-CO4 and DR12-CO5, Fig. B.6) point away from the center of OB2 and are associated with the bright mid-IR filament extending southward from the DR15 $\mathrm{H}$ in region. That the mid-IR emission detected by MSX clearly outlines the two main CO clumps demonstrates a link between the DR15 region and this part of the DR12 complex.

The DR6 radio continuum source is embedded within the high-intensity CO clump DR6-CO1 (Fig. B.4). This H II region is created by the cluster [LK2002]Cl-05 ([DB2001]Cl-07), which contains at least 6 B0-O9.5 stars (Odenwald et al. 1986). West of DR6, a large-scale, rather diffuse V-shaped CO complex (indicated as DR6W in Fig. 8) seems to be shaped by a northern source that could be the OB9/IC $1318 \mathrm{~b} / \mathrm{c}$ cluster. Both DR6$\mathrm{CO} 1$ and DR6-CO2 are not directly correlated as outlined in Sect. 4.2.2.

The $\mathrm{H}_{\text {II }}$ region DR9 lies at the border of a compact clump (DR9-CO1, Fig. B.5) which is rather isolated and not 
linked to other $\mathrm{CO}$ emission in this region (e.g., molecular clouds associated with DR13, DR6). DR9 hosts the OB cluster [LK2002]Cl-04 ([DB2001]Cl-06) that is responsible for the strong mid-IR emission visible in the MSX image.

The large DR13 CO filament (see Fig. B.6) seems to be shaped by a source in the north or to the north west of the cloud, which could be Cygnus OB9 and/or OB2 or an unidentified embedded source. It shows strong mid-IR emission in the northern part of the complex. In particular, a bright rim feature is visible that is due to the OB cluster[LK2002]CL-06 ([DB2001]CL-08). More diffuse mid-IR emission is found around the $\mathrm{H}$ II region DR13 and the OB cluster [LK2002]Cl-07 ([DB2001]Cl-09). The bulk of ${ }^{13} \mathrm{CO}$ emission further south is not correlated to mid-IR emission.

\subsection{The W75N/AFGL2620, DR17, DR18, and DR20NW clouds: $v_{\mathrm{CO}}$ from +6 to $+20 \mathrm{~km} \mathrm{~s}^{-1}$}

In the CygX-North region (Fig. 9), we find an arc of mid-IR emission associated with the molecular clouds of the extended $\mathrm{H}$ II region DR17. The possibility that this feature represents a supernova remnant was discussed - and ruled out - by WardThompson \& Robson (1991). It is moreover likely that the stars powering the DR17 $\mathrm{H}$ II region are also responsible for heating the surrounding dust. The high brightness of the loop suggests a large number of $\mathrm{OB}$ stars at the center of the $\mathrm{H}$ II region. Recently, two OB clusters have been recognized in the 2MASS images [LK2002]Cl-12 ([DB2001]Cl-15) which could actually be the exciting source for the DR17 $\mathrm{H}$ II region and the mid-IR loop emission.

Another prominent feature in the KOSMA ${ }^{13} \mathrm{CO}$ emission map (not as strong in mid-IR emission) are the DR17 pillars (clumps DR17-1, 2, 3, 4 in Fig. B.1), which closely resemble molecular "pillars" such as those seen in the Carina (Rathborne et al. 2004) or Eagle nebula (Pound 1998). We suggest that they are shaped locally by the OB stars in the DR17 region and are associated with the DR17 loop/H II region. In addition, we note that the W75N/AFGL2620/DR17N clouds are also most probably part of the same complex since the shape of these clouds also points towards an influence from a UV source in the region of DR17. Also, if one extends the portion of the loop seen in the MSX image to a full circle, these three regions would more or less fall on that circle.

A strong mid-IR emission source is the stellar cluster [LK2002]Cl-13 ([DB2001]Cl-16) (the "Diamond Ring", Marston et al. 2004). Their Spitzer IRAC images reveal a filamentary structure, which is also visible in the MSX image at lower angular resolution (Fig. 9). The cluster is embedded in a prominent, nearly circular ${ }^{13} \mathrm{CO}$ clump (DR21-CO9, Fig. B.3). Another example of such an embedded cluster is $\mathbf{W 7 5 N}$, which appears equally prominent in mid-IR and ${ }^{13} \mathrm{CO}$ emission (clump DR21-CO10, Fig. B.3). The cluster members are late to earlytype B protostars (Sheperd et al. 2003) and produce massive molecular outflows.

Two other well-correlated ${ }^{13} \mathrm{CO} / \mathrm{mid}-\mathrm{IR}$ emission features are seen in this velocity range: the two small molecular clumps with a globular shape close to DR18 (DR18-CO1 in Fig. B.2), and DR20 (DR20-CO3 in Fig. B.2) both hosting IR sources. We conclude that they are shaped by the influence of the Cygnus OB 2 cluster since they have an illuminated tip facing the cluster and a tail pointing away from its center.

\subsection{DR22 and DR23: $v_{\mathrm{CO}}$ from +6 to $+20 \mathrm{~km} \mathrm{~s}^{-1}$}

The ${ }^{13} \mathrm{CO}$ emission of the clouds close to DR22 (clump DR22CO5 in Fig. B.3) and DR23 (clumps DR23-CO3 and 4 in Fig. B.3) is not correlated with mid-IR emission although DR23-CO3 contains an MSX source. Moreover, the clumps seen in this velocity range appear detached from the large $\mathrm{CO}$ complex in the north (W75N/AFGL2620/DR17N).

\subsection{The DR15/DR7S arc complex: $v_{\mathrm{Co}}$ from +3 to $+20 \mathrm{~km} \mathrm{~s}^{-1}$}

The most prominent large-scale feature in this velocity range (Fig. 9) is an arc of $\mathrm{CO}$ emission consisting of the molecular cloud complexes DR7 and DR15. The arc seems to be shaped by Cygnus OB2 since the center of it is close to the center of OB2. Clumps DR7-CO5-9 (Fig. B.4) and clumps DR15-CO2-4 (Fig. B.6) are part of the arc. Just south of DR7 and 20' north west of DR15, the elongated clump DR7-CO5 is associated with a weak rim of mid-IR emission, suggesting an external influence from the south west (OB1-OB9 direction). Similar brightrimmed CO clumps (DR15-CO3 and DR15-CO2, hosting an IR-source; IRAS 20293+3952, FIR2 in Odenwald et al. 1990; Sridharan et al. 2002) seem to be influenced from the west (OB1OB9 direction). In the southeast part of the arc and east of the position of the $\mathrm{H}$ II region DR15, the clump DR15-CO4 is shaped by UV radiation, but the direction of the influence points north east towards OB2 instead of OB1-OB9 (see Sect. 5.3). Finally, in the far eastern part of the arc, more diffuse $\mathrm{CO}$ emission does not seem to be strongly influenced by any external UV radiation.

\section{Discussion}

\subsection{The distances in Cygnus $X$}

The distance determination to individual clouds and star-forming regions in Cygnus X has been a long-standing issue that is still largely unresolved. The reason for this is that kinematic distances cannot be used due to the degeneracy of the Galactic velocities in that direction (Galactic longitudes close to $90^{\circ}$ ). We illustrate this problem below with the example of the clouds directly influenced by Cygnus OB2 (Sect. 6.1.1) and discuss (Sect. 6.1.2) the distances in Cygnus $\mathrm{X}$ used in the literature.

\subsubsection{Velocity dispersion and kinematic distances around OB2}

From a comparison between ${ }^{13} \mathrm{CO}$ and mid-IR emission shown in Figs. 7 to 9 and the channel maps of Figs. 3 and 5, it is clear that a number of molecular clumps at very different velocities are affected by, therefore associated with, the Cyg OB2 cluster:

- Clump DR20-CO3 at $+13 \mathrm{~km} \mathrm{~s}^{-1}$ is a dense, compressed, cometary-shaped molecular cloud obviously exposed to the stellar winds and the UV radiation of the massive stars of OB2. It was argued by Odenwald et al. (1990) that OB2 cannot be responsible for triggering star formation in DR20 since the cluster center is too far away and the transit time for shock propagation (a shock velocity of $10 \mathrm{~km} \mathrm{~s}^{-1}$ was assumed) is longer than the age of the cluster (they used $10^{6} \mathrm{yr}$ and a distance to OB2 of $2-3 \mathrm{kpc}$ ). But with a distance of $1.7 \mathrm{kpc}$ and an age of $5 \times 10^{6} \mathrm{yr}$ for OB2 (Uyaniker et al. 2001 ), the ionization front propagated $50 \mathrm{pc}$ and thus could have already reached DR20. (See, e.g., Figs. 7 to 9 where a 
circle of $30 \mathrm{pc}$ radius is drawn around OB2.) Using the same numbers, direct compression of gas due to expanding ionization fronts is even possible for a number of other sources in the region, including AFGL2591 (see below).

- Clump DR18-CO1 at $+13 \mathrm{~km} \mathrm{~s}^{-1}$ shows a globular shape pointing away from the OB2 cluster center. Comeron \& Torra (2001) used their near-IR observations to deduce that the DR18 H II region is shaped on a small scale by a loose aggregate of stars with one luminous B0.5V star at a distance of $1.6 \mathrm{kpc}$. However, the observed extended and directed (towards OB2) mid-IR emission seen with MSX requires an even stronger influence, which can only be due to Cyg OB2.

- The globules DR7-CO1 and DR7-CO2 south west of the OB2 cluster center at velocities -11 and $-4 \mathrm{~km} \mathrm{~s}^{-1}$, the YSO AFGL2591 $\left(-8 \mathrm{~km} \mathrm{~s}^{-1}\right)$, and the clumps DR12-CO4,5 and DR15-CO4,5 all show evidence of also being shaped by the influence of the stellar winds and radiation of the massive stars of OB2.

All these clouds show clear interactions with Cyg OB2 and thus must be at the same distance, while their $\mathrm{CO}$ velocities vary from -11 to $+13 \mathrm{~km} \mathrm{~s}^{-1}$, which would represent a spread in kinematic distances of 0 to $5 \mathrm{kpc}$. This also shows how large $\left(24 \mathrm{~km} \mathrm{~s}^{-1}\right)$ the velocity dispersion can be around an OB association as massive as Cyg OB2 (see Sect. 6.4).

\subsubsection{Other distance estimates in Cygnus $X$}

It was recognized by Ikhsanov (1959) and Véron (1965) that a large number of $\mathrm{H}$ II regions in Cygnus $\mathrm{X}$ appear to lie at a canonical distance of about $1.5 \mathrm{kpc}$, leading those authors to propose that the Cygnus $\mathrm{X}$ direction is dominated by a single, large, and very active star-forming complex at a distance close to $1.5 \mathrm{kpc}$. Dickel et al. (1969) discussed this conclusion again by pointing out that these early works based on optical data could have been biased toward the less extincted and therefore less distant $\mathrm{H}$ II regions. Dickel et al. derived visual extinctions toward $90 \mathrm{H}$ II regions. Assuming that the extinction is a pure linear function of distance toward Cygnus X, they derived the distances of these $\mathrm{H}_{\text {II }}$ regions. As a result of this approach, they proposed that the objects in Cygnus $X$ are at distances ranging from 1.2 to $\sim 4 \mathrm{kpc}$. However, toward a network of GMCs such as Cygnus X, the extinction is not a smooth function of the distance, but is low until the edges of the clouds and then suddenly increases inside the molecular clouds. Therefore, whenever an $\mathrm{H}$ II region is located behind or inside a cloud, the extinction towards this object can easily increase by 5 to 10 mag over a relatively short distance, typically a few pc, i.e., the typical size of a cloud. In such a case, in the interpretation of Dickel et al., this $\mathrm{H}_{\text {II }}$ region is placed at a large distance, corresponding to the increase in extinction if it were a steady function of distance. In fact, the histogram of the distances in Dickel et al. is bimodal (see their Fig. 3), with $2 / 3$ of the regions lying between 1.2 and $2.4 \mathrm{kpc}$ and $1 / 4$ at significantly larger distances (more than $3.8 \mathrm{kpc}$ ). Such a bimodal distribution is exactly what one expects if a fraction of the $\mathrm{H}$ II regions are either embedded inside or located behind molecular clouds that locally increase their extinction along the line of sight. For these clouds, the distances derived by Dickel et al. will be heavily overestimated, leading to the spread up to $\sim 4 \mathrm{kpc}$ in their distribution.

More recently, this "extinction wall" at a distance of $\sim 1.5 \mathrm{kpc}$ was observed again by Straizys et al. (1993), who used spectroscopy of foreground stars in the directions of the North America nebula and the main Cygnus X region. Toward the North America nebula, the extinction starts at a distance of $\sim 500$ pc. Toward the main Cygnus $X$ region, in contrast, there is an indication of a relatively high transparency for the material up to $\sim 1.5 \mathrm{kpc}$ (see Figs. 4 and 5 of Straizys et al. 1993).

After these early attempts to derive distances to $\mathrm{H}$ in regions, only a few additional objects in Cygnus $\mathrm{X}$ had distances assigned that were often afflicted with large uncertainties. A summary of distance estimates for Cygnus X objects can be found in Uyaniker et al. (2001). Three OB associations (Cyg OB1, OB2, and OB9) and one SN remnant (DR4) are located inside our mapped region and are relevant to our distance discussion. Interestingly enough, their distances are all between 1.2 and $1.7 \mathrm{kpc}$, with the most accurate distance being $1.7 \mathrm{kpc}$ for Cyg OB2 (Massey \& Thompson et al. 1991). Our detailed analysis of the $\mathrm{CO}$ emission toward Cygnus $\mathrm{X}$ newly indicates the existence of at least a few large, homogeneous CO complexes that allow us to revisit the long-standing distance issue for Cygnus X.

\subsection{Coherent groups of clouds and their distances}

Because kinematic distances are not reliable toward Cygnus X, $\mathrm{CO}$ velocity-position patterns and the association of clouds with PDR features in MSX images provide important proofs of coherent groups of clouds and their association with stellar groups. The global problem of distances in Cygnus $X$ therefore reduces to the problem of the distances to only a few groups of clouds.

Essentially all CO clouds are recognized as part of only 5 groups as defined in Sects. 4 and 5 and can be seen in the position-velocity plot of the whole Cygnus X region (Fig. 10). This plot shows an average of all ${ }^{13} \mathrm{CO} 2 \rightarrow 1$ emission between Galactic latitudes $\sim-1^{\circ}$ and $\sim+2^{\circ}$, i.e., the mapping area.

The Group I: DR20, DR21, DR22, and DR23 appear in Fig. 10 as a coherent feature from $l=80.5^{\circ}$ to $82^{\circ}$ with $v_{\mathrm{CO}}=-7$ to $+1 \mathrm{~km} \mathrm{~s}^{-1}$ comprising DR22, DR20, DR23, and DR21. This rather massive $\left(167000 M_{\odot}\right)$ group hosts a number of young embedded clusters and protostars but does not show any definite association with one of the $\mathrm{OB}$ associations of the region.

The Group II: W75N, DR17, GL2620 emerges in Fig. 10 as mostly 3 massive $\left(54500 M_{\odot}\right)$ clouds from $l=81^{\circ}$ to $82^{\circ}$ with $v_{\mathrm{CO}}=+6$ to $+20 \mathrm{~km} \mathrm{~s}^{-1}$ comprising W75N, GL2620, and DR17. These 3 clouds have been recognized as obviously influenced by an unknown source situated close to DR17 (see Sect. 5), which is probably related to the prominent large loop of DR17 already discussed by Ward-Thompson \& Robson (1991).

From absorption studies of $\mathrm{OH}$ and $\mathrm{H}_{2} \mathrm{CO}$ (Dickel \& Wendker 1978), it emerges that W75N lies in front of DR21. A direct link to the DR21 clouds is then possible, as was suggested by Dickel et al. (1978), who assumed that DR21 and W75N are interacting molecular cloud complexes. We exclude the possibility that the whole W75N/AFGL2620/DR17N complex and the DR17 pillars are too close $(<1 \mathrm{kpc})$, because they do not appear prominent (high $A_{\mathrm{v}}$ ) in our extinction map (see Bontemps et al. 2006, in prep.).

The Group III: OB2 comprises all clouds that show a clear and direct influence from Cygnus OB2, i.e. clumps DR7-CO1-4, DR12-CO4,5, DR15-CO4,5, DR18-CO1, DR20CO3,4 and AFGL2591. As discussed in the previous section, it covers a wide range of velocities with $v_{\mathrm{CO}}=-11$ to $+13 \mathrm{~km} \mathrm{~s}^{-1}$ and a total mass of $37000 M_{\odot}$.

The Group IV: DR4,5,9,12,13,15 is the largest and most massive $\left(420000 M_{\odot}\right)$ group. In Fig. 10, it covers $l=77.4^{\circ}$ to $80^{\circ}$ with $v_{\mathrm{CO}}=-5$ to $+3 \mathrm{~km} \mathrm{~s}^{-1}$. Its homogeneous appearance supports the idea that it is actually a single complex. Some strong indications of influence from both Cyg OB2 and Cyg OB1/OB9 


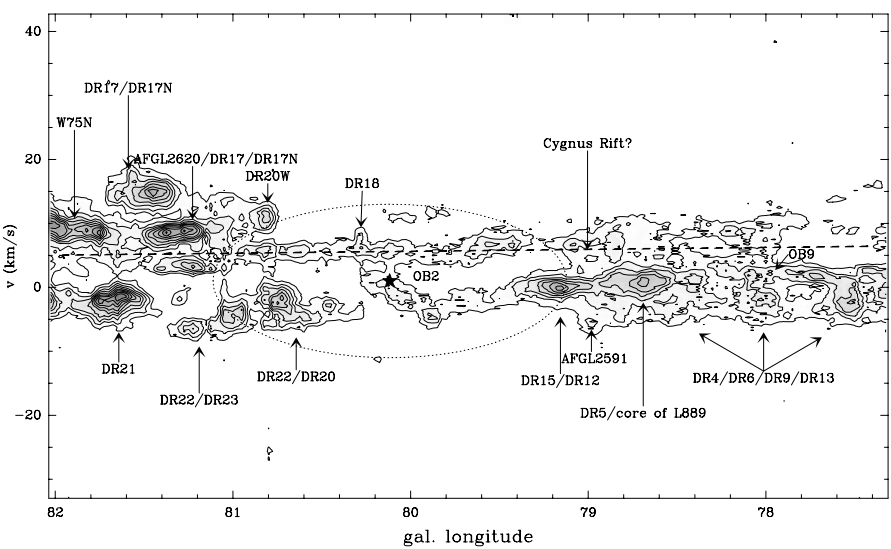

Fig. 10. Position-velocity cut in ${ }^{13} \mathrm{CO} 2 \rightarrow 1$ averaged over Galactic latitudes $\sim-1^{\circ}$ to $\sim 2^{\circ}$. Maps of ${ }^{13} \mathrm{CO}$ emission in the whole Cygnus $\mathrm{X}$ region in Galactic coordinates are found in Figs. 7 to 9. The minor axis of the dotted ellipse is defined by the highest and lowest velocities of globules $\left(13 \mathrm{~km} \mathrm{~s}^{-1}\right.$ and $\left.-11 \mathrm{~km} \mathrm{~s}^{-1}\right)$ symmetrically around $+1 \mathrm{~km} \mathrm{~s}^{-1}$. The major axis is given by the diameter of the OB2 cluster $\left(2^{\circ}\right.$, Knödelseder et al. 2000). The center is defined by the longitude of the OB2 cluster center $\left(80.12^{\circ}\right.$, Knödelseder et al. 2000) and by $1 \mathrm{~km} \mathrm{~s}^{-1}$ in velocity space (see above). The dashed line indicated the possible velocity component of the Cygnus Rift, considering a distance of $800 \mathrm{pc}$ and using the Galactic rotation curve.

have been discussed in Sect. 5. The clouds of Group IV could therefore be lying between these two stellar groups.

The last group, referred to as Group V, corresponds to diffuse $\mathrm{CO}$ clouds that could not be clearly recognized as part of the well-defined features discussed in Sects. 4 and 5. They include a few clouds from DR17, DR17N, and DR12 and appear in Fig. 10 as a probable single feature covering the whole range of Galactic longitudes but with a rather narrow velocity range $v_{\mathrm{CO}}=+3$ to $+7 \mathrm{~km} \mathrm{~s}^{-1}$ that is very close to the expected CO velocity of nearby gas. We therefore interpret this feature as the Cygnus Rift clouds that are believed to be at a distance of 600-800 pc from Sun. The total mass would then be $\sim 10000 M_{\odot}$.

Finally, only a few compact CO clouds do not show any clear association with one of the groups. DR23-CO3,4 spatially coincides with the DR23 complex and could actually be part of it despite the large velocity difference of $15 \mathrm{~km} \mathrm{~s}^{-1}$ between these two parts of DR23. DR22-CO5 is quite similar to the case above. This cloud is spatially coincident with the western part of the DR22 region with a velocity difference of $\sim 11 \mathrm{~km} \mathrm{~s}^{-1}$. Finally, DR6-CO1 is a rather compact CO cloud associated with the DR6 $\mathrm{H}$ II region and does not show any sign of being associated with the surrounding molecular material. It could therefore be a completely separate cloud. Its compactness may indicate that it is at a larger distance than the rest of Cygnus X. From its $\mathrm{CO}$ velocity $\left(v_{\mathrm{CO}}=-2.6 \mathrm{~km} \mathrm{~s}^{-1}\right)$, it cannot be part of the Perseus arm, but it can still be as far away as $\sim 3 \mathrm{kpc}$.

\subsection{The nature of Cygnus $X$}

The discussion of the nature of Cygnus $\mathrm{X}$ has remained controversial since the earliest observations of this region. There are two different scenarios:

1. Cygnus $X$ is a large Strömgren sphere surrounding the Cyg OB2 association (Véron 1965; Landecker 1984; McCutcheon \& Shuter 1970). In this case, the majority of the objects are located at approximately the same distance, i.e., that of the Cyg OB 2 cluster, which is rather safely determined to $1.7 \mathrm{kpc}$ (e.g., Knödlseder 2000). In this scenario, the sources ( $\mathrm{H}_{\text {II }}$ regions, molecular clouds, etc.) can be located at the front and the back of a wind-blown cavity created by OB 2, possibly forming an expanding shell. This view is not exactly identical to the so-called "superbubble" scenario described by Cash et al. (1980) and Uyaniker et al. (2001). The "Cygnus super bubble" (CSB) is an extended $\left(18^{\circ} \times 13^{\circ}\right)$, strong, soft X-ray emission region that is produced by the combined effect of supernova explosions and stellar winds from a number of OB associations.

2. The other view (favored by, e.g., Dickel et al. 1969; Piepenbrink \& Wendker 1988; Wendker et al. 1991) suggests that the large number of objects seen in Cygnus $\mathrm{X}$ is a chance superposition of star formation sites because the local spiral arm is seen tangentially. All features observed in Cygnus $\mathrm{X}$ would then be located at distances between $\sim 800 \mathrm{pc}$ and $4 \mathrm{kpc}$.

Our observations and analysis clearly favor the first interpretation. We summarized in Sect. 6.2 how the various molecular cloud complexes seen in Cygnus $\mathrm{X}$ are related both to each other and to the Cyg OB2 and OB9 star clusters. We think - thus conforming to views of, e.g., Landecker (1984) or Véron (1965) - that basically all massive molecular clouds seen now in Cygnus $\mathrm{X}$ form a coherent cloud complex out of which Cyg OB2, OB9, and possibly OB1 formed. The two major cloud complexes are CygX-North and CygX-South, or DR21 and IC 1318 in the nomenclature of Cong (1977), who already suggested that the molecular clouds and their associated $\mathrm{H}$ II regions form two related groups at similar distances. A similar two-peaked geometry was seen, e.g., in thermal continuum emission (Wendker 1970) and in the H166 $\alpha$ recombination line (Landecker 1984), which points toward an extended, probably expanding Strömgren sphere mainly created by Cyg OB2. Piepenbrink and Wendker (1988) excluded such an "expanding shell" scenario since the velocity distribution of this configuration is not observed. We do not, however, expect a simple velocity structure due to the influence of OB9 and the large number of secondary star formation sites triggered by OB2, OB9, and OB1. These star formation sites are evident as more or less evolved $\mathrm{H}_{\text {II }}$ regions (radio continuum sources DR7 to DR23) and (deeply embedded) IR-clusters. For example, we conclude that there could be clusters close to the DR13 and DR17 H II regions that have not yet been recognized (see Sects. 5.3 and 5.4). But in any case, it is evident that star formation is progressing into the clouds surrounding the OB2 association.

The large number and different generations of star formation sites complicate the whole scenario seen in radio and molecular line emission due to blending. The same is valid for observations of $\mathrm{H}_{\mathrm{I}}$ emission, which are additionally hampered by the ubiquitous distribution of neutral hydrogen. However, McCutcheon $\&$ Shuter (1970) suggest that the radio sources are distributed symmetrically with respect to $\mathrm{H}_{\mathrm{I}}$ and that they form a loosely associated group of clouds at a common distance. In an upcoming paper (Schneider et al. 2006, in prep.), we will use H I $21 \mathrm{~cm}$ data from the $\mathrm{CGPS}^{6}$ towards the Cygnus $\mathrm{X}$ region to obtain an overview of the emission distribution of atomic hydrogen and specifically to study $\mathrm{H}_{\text {I }}$ self-absorption (HISA) against diffuse $\mathrm{H}_{\mathrm{I}}$ background emission and $\mathrm{H}_{\mathrm{I}}$ absorption against radio continuum emission from $\mathrm{H}$ II regions. In certain directions, this will allow us to determine the line-of-sight distribution and properties of $\mathrm{H}$ I gas. A comparison between $\mathrm{H}$ I and $\mathrm{CO}$ line emission can then help to disentangle the individual features (H I cloud,

\footnotetext{
${ }^{6}$ Canadian Galactic Plane Survey.
} 
GMC, H II region) relative to each other along the line of sight (Kuchar \& Bania 1994).

So far, the relative positioning of the cloud complexes in Cygnus $\mathrm{X}$ remains difficult. The more diffuse emission distribution, lower extinction, and obvious associated foreground features, such as L889 and the IRDC's close to DR15, suggest that the CygX-South region is closer to the observer than the CygX-North region. This view is supported by Landecker (1984) who claims that CygX-South (North) is on the near (far) side of Cyg OB 2 and that ionized gas is streaming towards (away from) the observer, which would explain the observed H166 $\alpha$ velocities and rather large linewidths. The relative distance of CygXNorth and South is probably on the order of 50-100 pc, i.e. the typical extent of GMCs in the Milky Way.

In either case, the molecular clouds of the CygX-North region are more clearly defined with a high contrast filamentary structure and appear more "compressed" with rather strong $\mathrm{CO}$ emission gradients. There are also more gravitationally bound clumps in this region compared to CygX-South (see Sect. C.2), and those clumps have the highest densities found in Cygnus X. To what extent that results from CygX-North being a region where the gas is more processed, being closer to Cyg OB2, or having primordially different properties cannot be concluded from the present study. These aspects will be investigated in upcoming papers analyzing higher angular resolution ${ }^{13} \mathrm{CO}, \mathrm{C}^{18} \mathrm{O}, \mathrm{CS}$, and $\mathrm{N}_{2} \mathrm{H}^{+}$data.

\subsection{The large velocity breadth of the $\mathrm{CO}$ emission in Cygnus $X$}

In Sects. 4.1.3 and 4.2.3, it becomes obvious that the coverage of $\mathrm{CO}$ emission in Cygnus $\mathrm{X}$ is rather large $\left(\simeq 30 \mathrm{~km} \mathrm{~s}^{-1}\right)$. This velocity dispersion is in the upper range of what was observed in other star-forming GMCs of similar mass, e.g. $26 \mathrm{~km} \mathrm{~s}^{-1}$ for Carina (Schneider \& Brooks 2004), $20 \mathrm{~km} \mathrm{~s}^{-1}$ for W49 (Simon et al. 2001), or $\simeq 17 \mathrm{~km} \mathrm{~s}^{-1}$ for W51 (Carpenter \& Sanders 1998). In contrast, a much quieter, basically non-star-forming cloud in the same mass class, G216-2.5 (Williams \& Blitz 1998), covers only $\sim 10 \mathrm{~km} \mathrm{~s}^{-1}$ in $\mathrm{CO}$ emission. Since in the theory of the turbulent support of molecular clouds (e.g. MacLow \& Klessen 2004, for an overview), supersonic turbulence controls star formation and is itself driven mainly by large-scale events like supernovae (SN) explosions, one would expect in first order no significant difference in the total $\mathrm{CO}$ velocity dispersion between G216-2.5 and the Cygnus X clouds (both have a low statistic on SN explosions). The observed higher velocity dispersion in Cygnus is thus most likely due to the intervention of more local energetic phenomena, i.e. the expanding ionization front and the stellar winds from the OB stars of Cyg OB2, OB1 and OB9. Cloud-cloud collisions can be important for the most massive clouds (Blitz 1990; Carpenter \& Sanders 1998) and Dickel et al. (1978) indeed propose that the DR21 and W75N clouds are interacting (see Sect. 6.2). In this scheme, a collision of Group I $\left(v=-7,+1 \mathrm{~km} \mathrm{~s}^{-1}\right)$ and Group IV $\left(v=-5,+3 \mathrm{~km} \mathrm{~s}^{-1}\right)$ with Group II $\left(v=+6,+20 \mathrm{~km} \mathrm{~s}^{-1}\right)$ would produce the observed velocity dispersion. In this scenario, Group III $(v=-11$, $+13 \mathrm{~km} \mathrm{~s}^{-1}$ ), would be the location and the remains of the first collision where Cyg OB 2 was formed.

\section{Summary}

We present an $11 \mathrm{deg}^{2}{ }^{13} \mathrm{CO} 2 \rightarrow 1$ survey and smaller maps of ${ }^{13} \mathrm{CO}$ and ${ }^{12} \mathrm{CO} 3 \rightarrow 2$ emission for the molecular clouds in the Cygnus $\mathrm{X}$ region obtained with the KOSMA $3 \mathrm{~m}$ submmradiotelescope. The main results of this study are summarized below.

1. The velocity-integrated $\left(-10\right.$ to $20 \mathrm{~km} \mathrm{~s}^{-1}$ ) ${ }^{13} \mathrm{CO} 2 \rightarrow 1$ map reveals that the molecular gas is gathered in two large complexes located in projection approximately northeast and southwest of the Cyg OB2 association (named CygX-North and CygX-South, respectively). The masses are $2.8 \times 10^{5} M_{\odot}$ for CygX-North and $4.8 \times 10^{5} M_{\odot}$ for CygX-South.

2. Channel maps and a position-velocity plot of ${ }^{13} \mathrm{CO} 2 \rightarrow 1$ emission show smooth velocity-position transitions for the molecular clouds of CygX-North and South.

3. The most important velocity ranges of ${ }^{13} \mathrm{CO} 2 \rightarrow 1$ emission are (i) -10 to $-1 \mathrm{~km} \mathrm{~s}^{-1}$, which represents the bulk emission range of DR21, the DR22-23 filament and AFGL2591; (ii) -3 to $3 \mathrm{~km} \mathrm{~s}^{-1}$, corresponding to the dark lane L889, DR13; and (iii) 6 to $20 \mathrm{~km} \mathrm{~s}^{-1}$, covering the emission range from W75N, the DR15-loop, and the pillars of DR17.

4. A comparison between the ${ }^{13} \mathrm{CO} 2 \rightarrow 1$ emission and a midIR image obtained from MSX reveals a close association between the two tracers. A number of clumps are identified as dense and compressed remains of molecular gas, exposed to the stellar winds and the UV radiation of the massive stars of Cyg OB2 and OB9. We calculate that the ionization front of Cyg OB2 has a radius of at least $50 \mathrm{pc}$ around the center of OB2. The MSX image is also used to recognize physical associations between (embedded) clusters or $\mathrm{H}$ in regions and the molecular clouds. This information is then used to define the $3 \mathrm{D}$ distribution of molecular gas in the direction of Cygnus X.

5. The excitation conditions of the molecular gas and the masses of the clumps are derived from the $\mathrm{CO}$ mapping, assuming a distance of $1.7 \mathrm{kpc}$. We identify a total of 91 clumps in the ${ }^{13} \mathrm{CO} 2 \rightarrow 1$ channel-maps. Their excitation temperatures lie between 10 and $30 \mathrm{~K}$, the clump radii are $1-8 \mathrm{pc}$, and the masses are a few hundred to several ten thousand $M_{\odot}$. We find that the average excitation temperature and density are different for CygX-North and South: $T_{\mathrm{ex}}=17.5 \mathrm{~K}(13.5 \mathrm{~K})$ and $n=1.7 \times 10^{3} \mathrm{~cm}^{-3}$ $\left(0.9 \times 10^{3} \mathrm{~cm}^{-3}\right)$ for CygX-North(South), respectively.

6. An analysis of the equilibrium state of the clouds reveals that the CygX-North and CygX-South regions show differences in their physical state. CygX-North seems to be a more active star forming region. A study of the variation in the physical properties (excitation temperature, mass, radius, and line width) of the clumps with distance to the OB cluster shows a weak tendency that the clump radii and line widths increase with distance to OB2.

7. We conclude that there is growing evidence from our new large-scale KOSMA data that the molecular clouds in Cygnus X form a coherent complex located at the same distance, i.e., that of the OB2/9 clusters at $\sim 1.7 \mathrm{kpc}$. In this picture, OB2 and OB9 represent the first generation of clusters formed within a large molecular cloud. Around OB2, a cavity nearly devoid of molecular gas is seen. In both regions, signposts of the formation of the next generation of clusters were discovered. It is possible that cloud-cloud collisions are responsible for the observed high velocity width $\left(30 \mathrm{~km} \mathrm{~s}^{-1}\right)$ of $\mathrm{CO}$ emission. Star formation, with Cyg OB2 as the first generation of stars, might have been triggered by these collisions.

Acknowledgements. The KOSMA $3 \mathrm{~m}$ submillimeter telescope at the Gornergrat-Süd is operated by the University of Cologne in collaboration with 
Bonn University and supported by special funding from the Land NRW. The observatory is administered by the International Foundation Gornergrat and Jungfraujoch.

This research made use of data products from the Midcourse Space Experiment. Processing of the data was funded by the Ballistic Missile Defense Organization with additional support from the NASA Office of Space Science. This research also made use of the NASA/ IPAC Infrared Science Archive, which is operated by the Jet Propulsion Laboratory, California Institute of Technology, under contract with the National Aeronautics and Space Administration.

This publication makes use of data products from the Two Micron All Sky Survey, which is a joint project of the University of Massachusetts and the Infrared Processing and Analysis Center/California Institute of Technology, funded by the National Aeronautics and Space Administration and the National Science Foundation.

\section{References}

Ackermann, G. 1970, A\&A, 8, 315

Alter, G., Balazs, B., \& Ruprecht, J. 1970, Catalog of Star Clusters and Associations (Budapest: Akademiai Kiado)

Baars, J. W. M., \& Wendker, H. J. 1981, A\&A, 101, 39

Bertoldi, F., \& McKee, C. 1992, ApJ, 395, 140

Blitz, L. 1990, in The evolution of the interstellar medium (Astronomical Society of the Pacific), 273

Bloemen, J. B. G. M., Strong, A. W., Mayer-Hasselwander, H. A., et al. 1986, A\&A, 154, 25

Bykov, A. M., Krassilchtchikov, A. M., Uvarov, M., et al. 2004, A\&A, 427, L21

Cambrésy, L., Beichman, C. A., Jarrett, T. H., \& Cutri, R. M. 2002, AJ, 123, 2559

Campbell, M. F., Hoffmann, W. F., \& Thronson, H. A. 1981, ApJ, 247, 530

Carpenter, J., M., \& Sanders, D. N. 1998, ApJ, 116, 1856

Cash, W., Charles, P., Bowyer, S., et al. 1980, ApJ, 238, L71

Clemens, D. P. 1985, ApJ, 295, 422

Comeron, F., \& Torra, J. 1999, A\&A, 349, 605

Comeron, F. \& Torra, J. 2001, A\&A, 375, 553

Cong, H. L. 1977, Ph.D. Thesis, Columbia University

Dame, T. M., Ungerechts, H., Cohen, R. S., et al. 1987, ApJ, 322, 706

Dickel, H. R., \& Wendker, H. J. 1978, A\&A, 66, 289

Dickel, H. R., Wendker, H. J., \& Bieritz, J. H. 1969, A\&A, 1, 270

Dickel, H. R., Seacord II, A. W., \& Gottesman, S. T. 1977, ApJ, 218, 133

Dickel, J. R., Dickel, H. R., \& Wilson, W. J. 1978, ApJ, 223, 840

Downes, D., \& Rinehart, R. 1966, ApJ, 144, 937

Dutra, C. M., \& Bica, E. 2001, A\&A, 376, 434

Egan, M. P., Shipman, R. F., Price, S. D., et al. 1998, ApJ, 494, L199

Elmegreen, B. G. 1998, ASP Conf. Ser., 148, 150

Goudis, C., \& Meaburn, J. 1974, A\&A, 34, 437

Graf, U. U., Haas, S., Honingh, C., et al. 1998, Proc. SPIE, 3357, 159

Grossmann, E. 1989, Atmosph. Transmission Software, User's Manual, Univ. of Texas, Austin

Ikshanov, R. N. 1959, Izv. Krymak. Astrofiz. Observ., 21, 229
Jakob, H., Kramer, C., Simon, R., et al. 2006, A\&A, accepted Knödlseder, J. 2000, A\&A, 360, 539

Kramer, C., Beuther, H., Simon, R., et al. 1999, ASP, CF-217

Kuchar, T. A., \& Bania, T. M. 1994, ApJ, 436, 117

Lada, C. J., Lada, E. A., Clemens, D. P., \& Bally J. 1994, ApJ, 429, 694

Landecker, T. L. 1984, AJ, 89, 1

Landecker, T. L., Roger, R. S., \& Higgs, L. A. 1980, A\&AS, 39, 13

LeDuigou, J.-M., \& Knödlseder, J., A\&A, 392, 869

Leung, H., \& Thaddeus, P. 1992, ApJS, 81, 267

Lombardi, M., \& Alves, J. 2001, A\&A, 377, 1023

Lynds, B. T. 1962, ApJS, 7, 1

MacLow, M.-M., \& Klessen, R. S. 2004, Rev. Mod. Phys., 76, 125

Maddalena, R., \& Thaddeus, P. 1985, ApJ, 294, 231

Mangum, T., Wootten, A., \& Mundy, A. 1991, ApJ, 378, 576

Marston, A. P., Reach, W. T., Noriega-Crespo, A., et al. 2004, ApJ, 154, 333

Massey, P., \& Thompson, A. B. 1991, AJ, 101, 1408

McCutcheon, W. H., \& Shuter, W. L. H. 1970, AJ, 75, 910

Motte, F., Bontemps, S., Schilke, P., et al. 2005, Proc. IAU Symp., 227 (Cambridge University Press), 151

Odenwald, S., Shivanandan, K., Campbell, M., et al. 1986, ApJ, 306, 122

Odenwald, S., Campbell, M., Shivanandan, K., et al. 1990, AJ, 99, 1

Oka, T., Yamamoto, S., Iwata, M., et al. 2001, ApJ, 558, 176

Piddington, J. H., \& Minnet, H. C. 1952, Australian J. Sci. Res., 5A, 17, 326, 339

Piepenbrink, A., \& Wendker, H. J. 1988, A\&A, 191, 313

Pound, M. W. 1998, ApJ, 493, L113

Rathborne, J. M., Brooks, K. J., Burton, M. G., Cohen, M., \& Bontemps, S. 2004, A\&A, 418, 563

Richardson, K. J., Sandell, G., Krisciunas, K., et al. 1989, A\&A, 224, 199

Robin, A. C., Reylé, C., Derrière, S., \& Picaud, S. 2003, A\&A, 409, 523

Schieder, R., Tolls, V., \& Winnewisser, G. 1989, Exp. Astron., 1, 101

Schneider, N., \& Brooks, K. 2004, PASA, 21, 290

Schneider, N., Simon., R., Kramer, C., et al. 2003, A\&A, 406, 915

Sheperd, D. S., Testi, L., \& Stark, D. P. 2003, ApJ, 584, 882

Simon, R., Jackson, J. M., Clemens, D. P., et al. 2001, ApJ, 551, 747

Sridharan, T. K., Beuther, H., Schilke, P., et al. 2002, ApJ, 566, 931

Straizys, V., Kazlauskas, A., Vansevicius, V., et al. 1993, Baltic Astronomy, 2, 171

Uyaniker, B., Fürst, E., Reich, W., et al. 2001, A\&A, 371, 675

van der Tak, F. F. S., van Dishoeck, E. F., Evans, N. J., \& Bakker, E. J. 1999, ApJ, 522, 991

Véron, P. 1965, AnAp, 28, 391

Ward-Thompson, D., \& Robson 1991, MNRAS, 248, 670

Wendker, H. J. 1970, A\&A, 4, 378

Wendker, H. J. 1984, A\&AS, 58, 291

Wendker, H. J., Schramm, K. J., \& Dieckvoss, C. 1983, A\&A, 121, 69

Wendker, H. J., Higgs, L. A., \& Landecker, T. L. 1991, A\&A, 241, 551

Westerhout, G. 1958, Bull. Astr. Inst. Netherlands, 14, 215

Wilson, T. L., \& Mauersberger, R. 1990, A\&A, 239, 305

Williams, J. P., \& Blitz, L. 1998, ApJ, 494, 657

Williams, J. P., Blitz, L., \& Stark, A. A. 1995, ApJ, 451, 252 
N. Schneider et al.: The Cygnus X region, Online Material $p 1$

\section{Online Material}




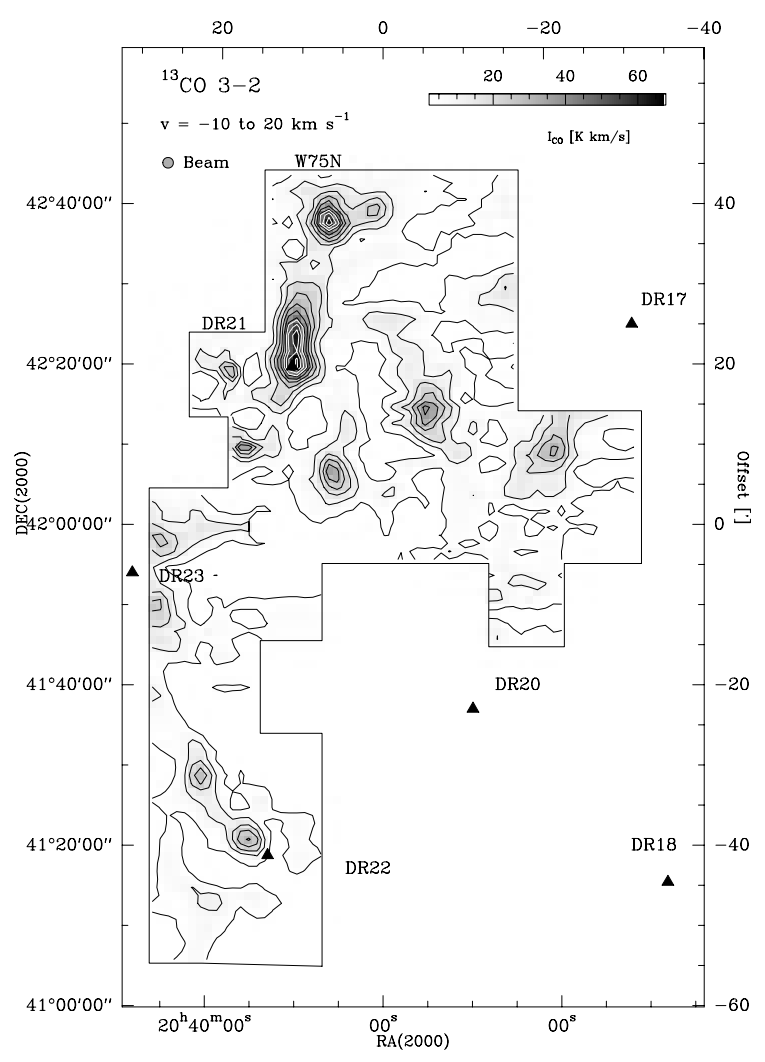

Fig. A.1. Line-integrated $\left(v=-10\right.$ to $\left.20 \mathrm{~km} \mathrm{~s}^{-1}\right){ }^{13} \mathrm{CO} 3 \rightarrow 2$ emission from the CygX-North region as observed with KOSMA. Contour lines are drawn in black from $3 \sigma$ to $57 \sigma$ in steps of $9 \sigma$ and in white from $60 \sigma$ to $87 \sigma$ in steps of $9 \sigma$ with $3 \sigma=2.3 \mathrm{~K} \mathrm{~km} \mathrm{~s}^{-1}$.

\section{Appendix A: Line integrated maps of ${ }^{13} \mathrm{CO}$ and ${ }^{12} \mathrm{CO} 3 \rightarrow 2$ emission}

The line integrated ${ }^{13} \mathrm{CO} 3 \rightarrow 2$ map of the CygX-North region, obtained in parallel to the ${ }^{13} \mathrm{CO} 2 \rightarrow 1$ line, is shown in Fig. A.1. This map is smaller because mainly winter weather conditions allow observations of the ${ }^{13} \mathrm{CO} 3 \rightarrow 2$ line. It misses the DR18, DR20, DR17N, and the eastern part of the DR23 region. The remaining map has a similar emission distribution like the ${ }^{13} \mathrm{CO} 2 \rightarrow 1$ data even though the emission peaks are more distinct, i.e. clearly showing up in the map. This is probably due to the higher critical density required for excitation of the $3 \rightarrow 2$ line that is found in the denser cloud interior. The outskirts of the cloud at lower density are traced by the ${ }^{13} \mathrm{CO} 2 \rightarrow 1$ line and even more in the low- ${ }^{12} \mathrm{CO}$ lines.

The line integrated ${ }^{12} \mathrm{CO} 3 \rightarrow 2$ map of the CygX-South region, also obtained in parallel to the ${ }^{13} \mathrm{CO} 2 \rightarrow 1$ line, is shown in Fig. A.2. This map covers only parts of the CygX-South region because the ${ }^{13} \mathrm{CO} 3 \rightarrow 2$ line was chosen in the high frequency band of the dual-channel receiver for the observations of the CygX-North region (see above). This map includes some major emission regions around DR6, DR7, DR12, and DR18, as well as clouds south of DR4 and DR13. It, however, misses the dark lane L889. The general appearance of this map is very similar to the ${ }^{13} \mathrm{CO} 2 \rightarrow 1$ map of the region (Fig. 2), although we observe that some molecular clouds split up into smaller fragments (e.g. DR13S). This is due to the higher angular resolution of the ${ }^{12} \mathrm{CO} 3 \rightarrow 2$ observations.

\section{Appendix B: Line integrated maps of ${ }^{13} \mathrm{CO} 2 \rightarrow 1$ emission in individual regions}

Figures B. 1 to B.6 show more detailed maps of line integrated ${ }^{13} \mathrm{CO} 2 \rightarrow 1$ emission of the molecular clouds in the Cygnus $\mathrm{X}$ region. The total area of all maps summed up covers the whole ${ }^{13} \mathrm{CO} 2 \rightarrow 1$ mapping region. Some figures show two velocity ranges, one with grey scaling and grey contours, the other one with only contours in which the line thickness increases with CO intensity. The dashed (blue) contours mark the $25 \%$ maximum intensity level of a clump that is used for the mass determination (see following section). Prominent sources (radio sources, OB clusters) are marked and named in the plots.

These figures are intended to serve as a finding chart for the clumps listed in Tables C.1 and C.2, as well as for the IR sources, OB clusters, and radio sources and for their association with individual clumps.

\section{Appendix C: Clump properties}

\section{C.1. Excitation conditions and masses derived from CO mapping}

In order to determine the excitation conditions throughout the whole Cygnus $\mathrm{X}$ region, we performed a straightforward LTE (local thermodynamic equilibrium) analysis, using ${ }^{12} \mathrm{CO}$ and ${ }^{13} \mathrm{CO} 2 \rightarrow 1$ and $3 \rightarrow 2$ data.

Assuming LTE and beam-filling factors of 1, the excitation temperature is determined in two ways:

(1) $T_{\text {ex12 }}$ is calculated by assuming an optically thick ${ }^{12} \mathrm{CO} 3 \rightarrow$ 2 or $2 \rightarrow 1$ line.

$$
\begin{aligned}
& T_{\mathrm{ex}}(32)=16.6 \times\left[\ln \left(16.6 / T_{\mathrm{mb}}(\mathrm{CO} 32)+0.036\right)\right]^{-1}[\mathrm{~K}] \\
& T_{\mathrm{ex}}(21)=11.06 \times\left[\ln \left(11.06 / T_{\mathrm{mb}}(\mathrm{CO} 21)+0.187\right)\right]^{-1}[\mathrm{~K}] .
\end{aligned}
$$

(2) $T_{\text {ex13 }}$ is derived by assuming optically thin ${ }^{13} \mathrm{CO} 3 \rightarrow$ 2 and $2 \rightarrow 1$ lines so that the line integrated ratio $R=\int T\left({ }^{13} \mathrm{CO} 3 \rightarrow 2\right) \Delta v / \int T\left({ }^{13} \mathrm{CO} 2 \rightarrow 1\right) \Delta v$ gives $T_{\mathrm{ex} 13}=$ $-15.88 / \ln (R / 2.25)$. Since we assume LTE, the excitation temperatures for all isotopomers are assumed equal. With the resulting opacities and the line integrated ${ }^{13} \mathrm{CO} 2 \rightarrow 1$ intensity, we derive the ${ }^{13} \mathrm{CO}$ column density, $\mathrm{H}_{2}$ column density, and masses as described in, e.g., Schneider et al. (2003).

The area for the mass determination was defined by polygons on a $25 \%$ level of the peak emission intensity in one of the velocity ranges given in Sects. 4.1.3 and 4.2.3. Even though this method ignores a finer velocity structure, it already gives a good estimate of the mass comprised in the largest clumps. The polygons define the boundaries of the clumps and are shown in Figs. B.1 to B.6. For easier identification, we divided the whole Cygnus $\mathrm{X}$ map into segments and named these after the corresponding radio source. This is a purely $2 \mathrm{D}$ selection but will be useful for recognizing and describing the different observational features. A distance of $1.7 \mathrm{kpc}$ was assumed for all mass estimates, which is probably not the exact value for all clumps (see Sect. 6). If a different distance $\mathrm{d}[\mathrm{kpc}]$ is applied, the masses have to be scaled by $(d / 1.7)^{2}$.

Tables C. 1 and C. 2 give an overview of the physical conditions of the bulk emission of the clumps found in the different velocity ranges in the CygX-North and CygX-South regions. The results at the position of DR21 should be treated with caution since the ${ }^{12} \mathrm{CO}$ line shows self-absorption effects 


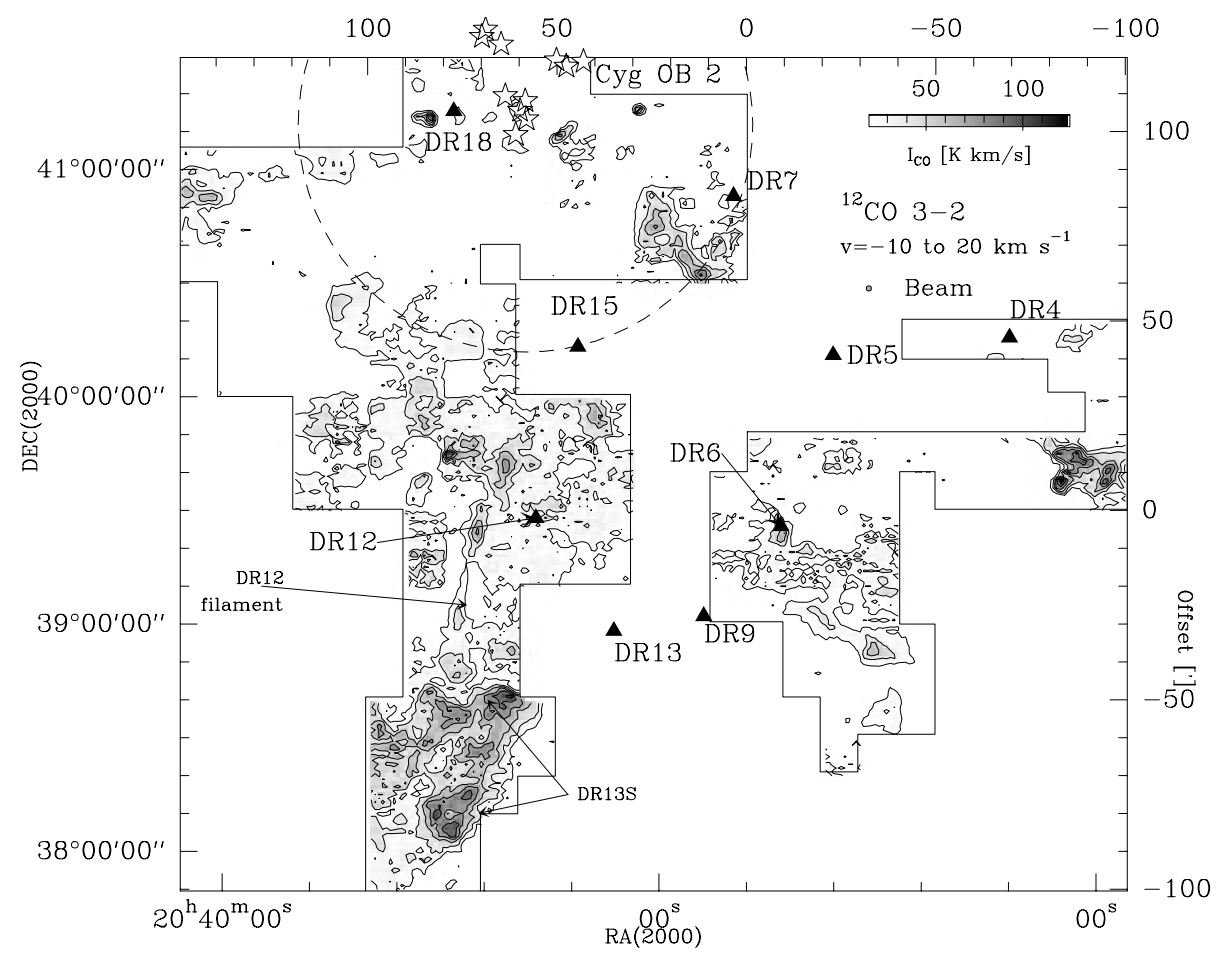

Fig. A.2. Line-integrated $\left(v=-10\right.$ to $\left.20 \mathrm{~km} \mathrm{~s}^{-1}\right){ }^{12} \mathrm{CO} 3 \rightarrow 2$ emission from the CygX-South region as observed with KOSMA. Contour lines are drawn in black from $15 \sigma$ to $75 \sigma$ in steps of $12 \sigma$ with $3 \sigma=4 \mathrm{~K} \mathrm{~km} \mathrm{~s}^{-1}$.

around $-5 \mathrm{~km} \mathrm{~s}^{-1}$. The excitation temperatures are therefore lower limits, and the opacities and column densities upper limits. The excitation temperature of the gas is typically around $20 \mathrm{~K}$, maximum values are found at DR21 ( 30 K), minimum values for DR5 $(\sim 7 \mathrm{~K})$. For the positions where we have the ${ }^{13} \mathrm{CO} 3 \rightarrow 2$ and $2 \rightarrow 1$ lines, we independently determined the excitation temperature using the line ratio (see above). The agreement is generally good so that our assumption of LTE is justified to a first order.

The opacity of the ${ }^{13} \mathrm{CO} 2 \rightarrow 1$ line is mainly smaller than 1 even though the lines are only marginally optically thin. The same holds for the ${ }^{13} \mathrm{CO} 3 \rightarrow 2$ line, where the optical depth (not shown in the Table) varies between 0.2 and 2.3 (at 3 positions in DR22 $1<\tau<2.3$ ). For all regions observed in ${ }^{12} \mathrm{CO}$, this line is optically thick as was derived from the ${ }^{12} \mathrm{CO} /{ }^{13} \mathrm{CO}$ line ratio, which is typically $2-3$. The radii of the clumps are typically a few pc, the masses range between $\sim 500$ and $60000 M_{\odot}$ with a typical value of a few $1000 M_{\odot}$. The average excitation temperature and the density are quite different for CygX-North and South. The clumps in CygX-North are generally warmer $\left(\left\langle T_{\mathrm{ex}}\right\rangle=17.5 \mathrm{~K}\right)$ and denser $\left(\left\langle n\left(\mathrm{H}_{2}\right)\right\rangle=1.7 \times 10^{3} \mathrm{~cm}^{-3}\right)$ than the clumps in CygX-South $\left(\left\langle T_{\mathrm{ex}}\right\rangle=13.5 \mathrm{~K},\left\langle n\left(\mathrm{H}_{2}\right)\right\rangle=\right.$ $\left.0.9 \times 10^{3} \mathrm{~cm}^{-3}\right)$. Each larger cloud complex, to first order defined by the regions listed in Tables C.1 and C.2, consists of several fragments with different ranges of column densities, masses and densities. Integrating over the whole area of a region and comparing that to the mass values of single clumps shows that $70 \%$ to $90 \%$ of the total mass is located within the clumps. The total mass from this calculation gives $2.8 \times 10^{5} M_{\odot}$ for CygX-North and $4.5 \times 10^{5} M_{\odot}$ for CygX-South.

\section{C.2. The equilibrium state of the clumps}

The clumps identified in Sect. C.1 may be in very different dynamical states. The more massive clumps may be in gravitational virial equilibrium, stabilized against collapse by internal thermal, turbulent, and magnetic pressure. If the masses of such clumps are higher than a critical mass, they are strongly selfgravitating, have central density enhancements, and may collapse. Alternatively, less massive clumps can be overpressured, in which case they are either short-lived, transient objects or confined by the turbulent pressure of the interclump medium.

Neglecting magnetic pressure, the equilibrium state can be characterized by the virial parameter $\alpha_{\mathrm{v}}$, defined as the ratio of kinetic to gravitational energy density (Bertoldi \& McKee 1992):

$\alpha_{\mathrm{v}}=\frac{1160 \sigma^{2} R}{M}$

with the radius $R$ [pc], mass $M\left[M_{\odot}\right]$, and the 1D velocity dispersion $\sigma\left[\mathrm{km} \mathrm{s}^{-1}\right]$ of the clump.

For values $\alpha_{\mathrm{v}} \simeq 1$, the clumps are in gravitational virial equilibrium, and clumps with $\alpha_{\mathrm{v}}>1$ are either transient or bound by external pressure in the ambient material, in which case they are stable.

We determined $\alpha_{\mathrm{v}}$ for all clumps identified in Cygnus $\mathrm{X}$ and show the virial parameter in Fig. C.1 as a function of mass. It turns out that more than half of the clumps (55 out of 91) are gravitationally bound, the rest are pressure-confined/transient. The gravitationally-bound clumps belong mainly to the CygXNorth region: all clumps of DR18, DR21, and DR23 have $\alpha_{\mathrm{v}}<1$. These are also the clumps with the highest gas densities, which is consistent with the picture of a self-gravitating, centrally condensed clump. The opposite behavior is observed for CygX-South, where less clumps are gravitationally bound. In numbers, $42 \%$ (58\%) of the clumps in CygX-North (South) are gravitationally bound and $36 \%(64 \%)$ of the clumps in CygX-North (South) have high virial parameters and are possibly pressure-bound. The difference in the dynamical equilibrium state of the clumps in CygX-North and South may indicate a different evolutionary state and/or star formation activity in the 
N. Schneider et al.: The Cygnus X region, Online Material $p 4$
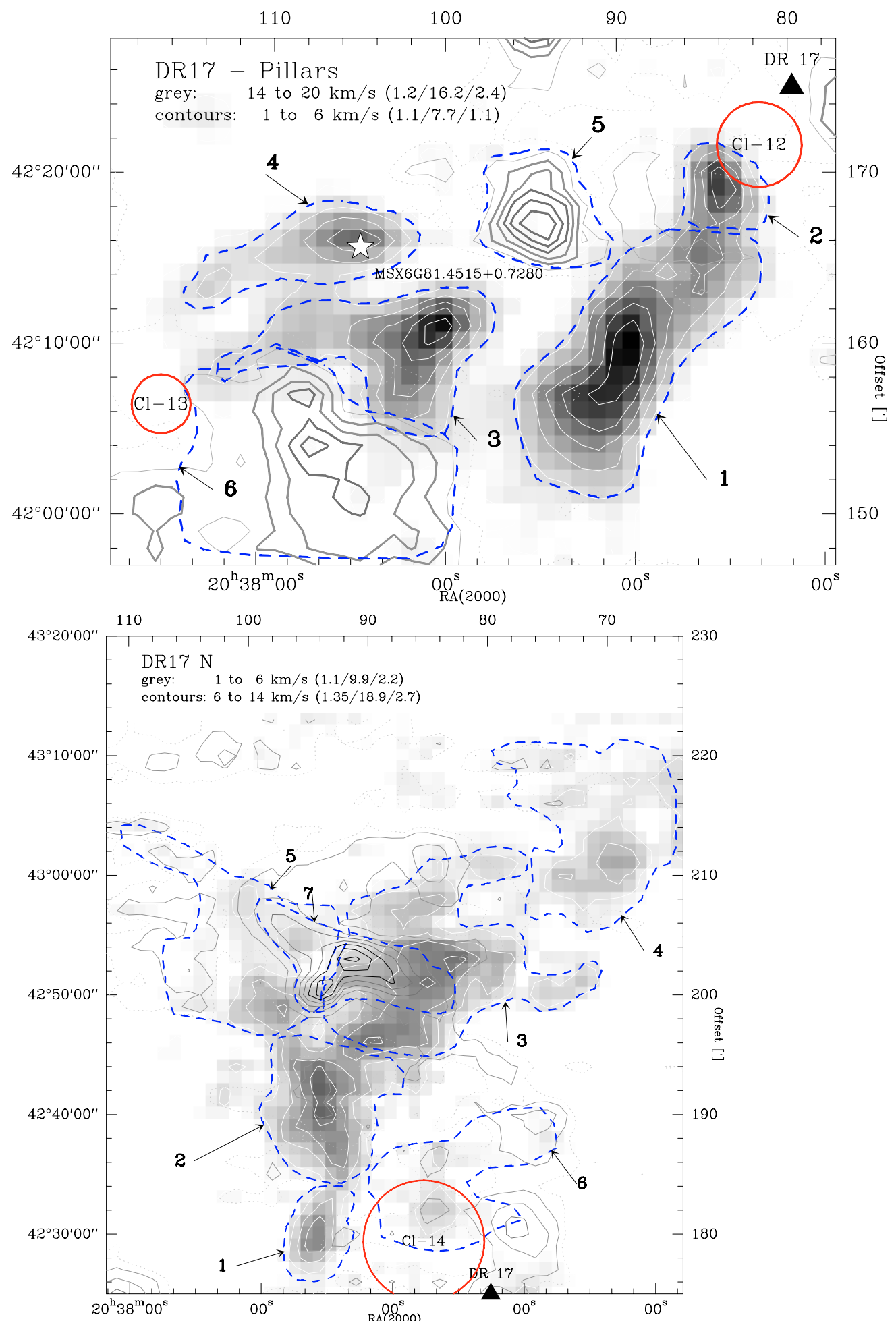

Fig. B.1. Velocity-integrated ${ }^{13} \mathrm{CO} 2 \rightarrow 1$ maps of the DR17 and DR17N regions in Cygnus X. Two velocity ranges are shown (one in grey scale and light contours and one with grey to black contours). The level notation is start/end/step with the values in $\mathrm{K} \mathrm{km} \mathrm{s}^{-1}$. All contours start at the $3 \sigma$ level. Dashed (blue) contours indicate the $25 \%$ maximum intensity level of a clump for the mass determination. Individual clumps are numbered according to Tables C.1 and C.2. Known IR-clusters are indicated by circles (size given by Le Duigou \& Knödlseder 2002) and IRAS and MSX sources by stars.

sense that the northern region is currently more actively forming stars, whereas the southern region is more quiescent.

We also note that nearly all clumps of the DR17 region (11 out of 13), which is most likely affected by an embedded cluster, and all "globules" that are shaped by OB2 (some clumps of DR7 and DR20) are overpressured and may only be confined by external pressure. This does not exclude the presence of collapsing cores, which may form stars, but they may also be completely transient features, i.e., being photo-evaporated by UV-radiation. 
N. Schneider et al.: The Cygnus X region, Online Material p 5

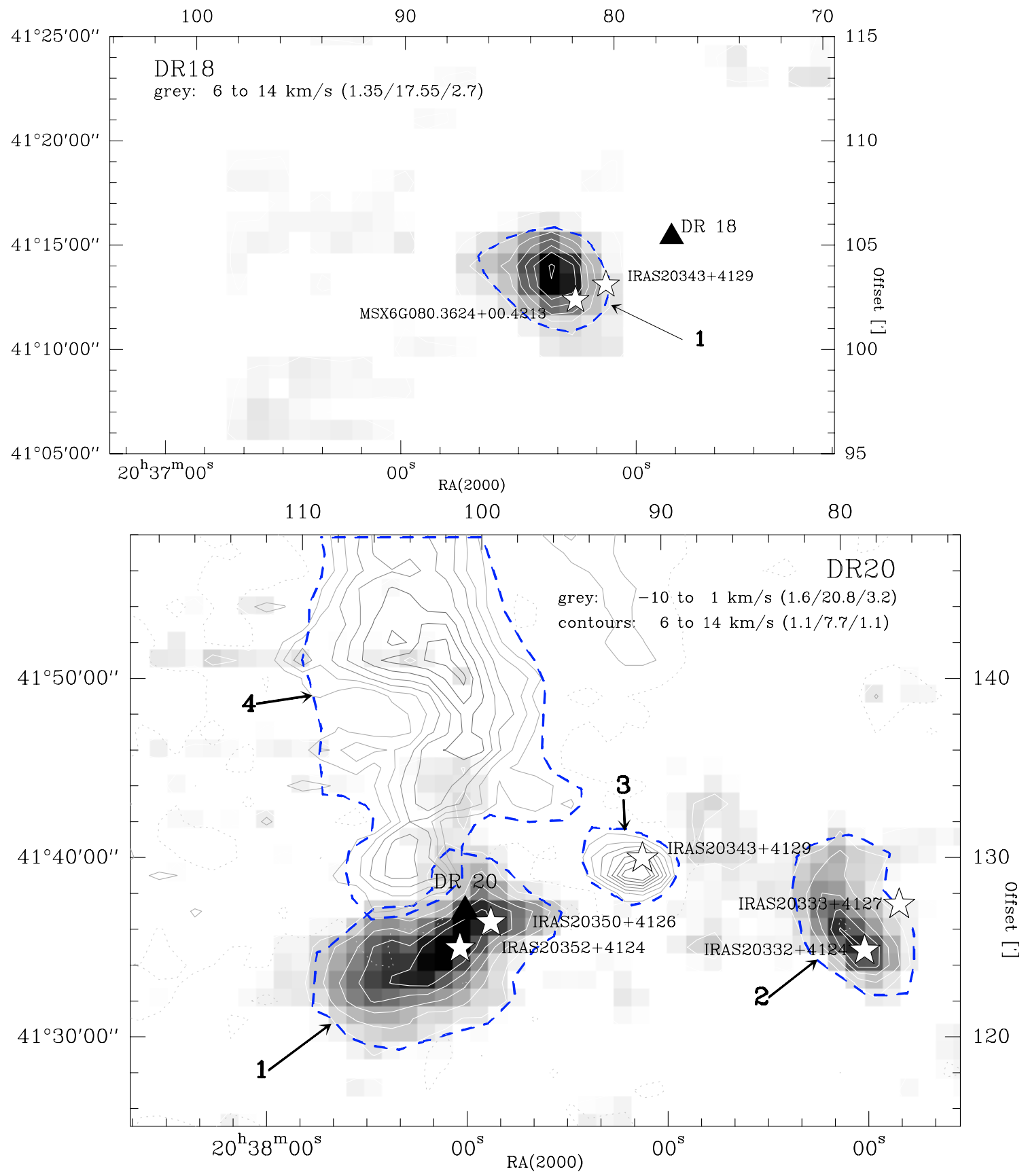

Fig. B.2. The same as Fig. B.1 but for the sources DR18 and DR20.

\section{C.3. Physical properties of the clumps and their variation across the complex}

Given the large number of examples seen in Cygnus $\mathrm{X}$ for the influence of the Cyg OB2 association on the molecular gas (see e.g. Sect. 5), it is motivating to investigate whether some physical properties of the clumps show a dependence as a function of projected distance to the cluster. We thus plotted the radius, mass, average $\mathrm{H}_{2}$-density, and line width of the clumps listed in Tables C.1 and C.1 against the distance to the Cyg OB2 cluster. For that, we assumed that all clumps are located at the distance of the OB 2 cluster. The radius may increase with distance which - since the density is nearly constant - causes the mass of the clumps to increase with distance as well. However, there is only a weak tendency by the clumps line width to increase with distance. Interestingly, this contrasts with a finding of Williams et al. (1995) for the Rosette molecular cloud, where only a gradient in excitation temperature and density with distance into the cloud was found due to the closely associated stellar OB cluster, complementary to our results.

Separating the results for the CygX-North and South regions shows no significant difference in properties even though the statistic is rather poor. However, it is mainly the more distant clumps that belong to the $\mathrm{CygX}$-South region that show this tendency toward larger radius and line width. A simple first conclusion could be that this is due to the fact that some of the farther clouds in CygX-South - assuming all objects are at the same distance - are less affected by the OB2 cluster. The DR21 region (at $v=-10$ to $1 \mathrm{~km} \mathrm{~s}^{-1}$ ) and the W75N/AFGL2620/DR17 region (at $v=6$ to $20 \mathrm{~km} \mathrm{~s}^{-1}$ ) mainly contain clumps with smaller radii. This category of clumps is mainly found close to the OB2 cluster in CygX-South (e.g. in the DR15 region at $v=3$ to $20 \mathrm{~km} \mathrm{~s}^{-1}$ ). 

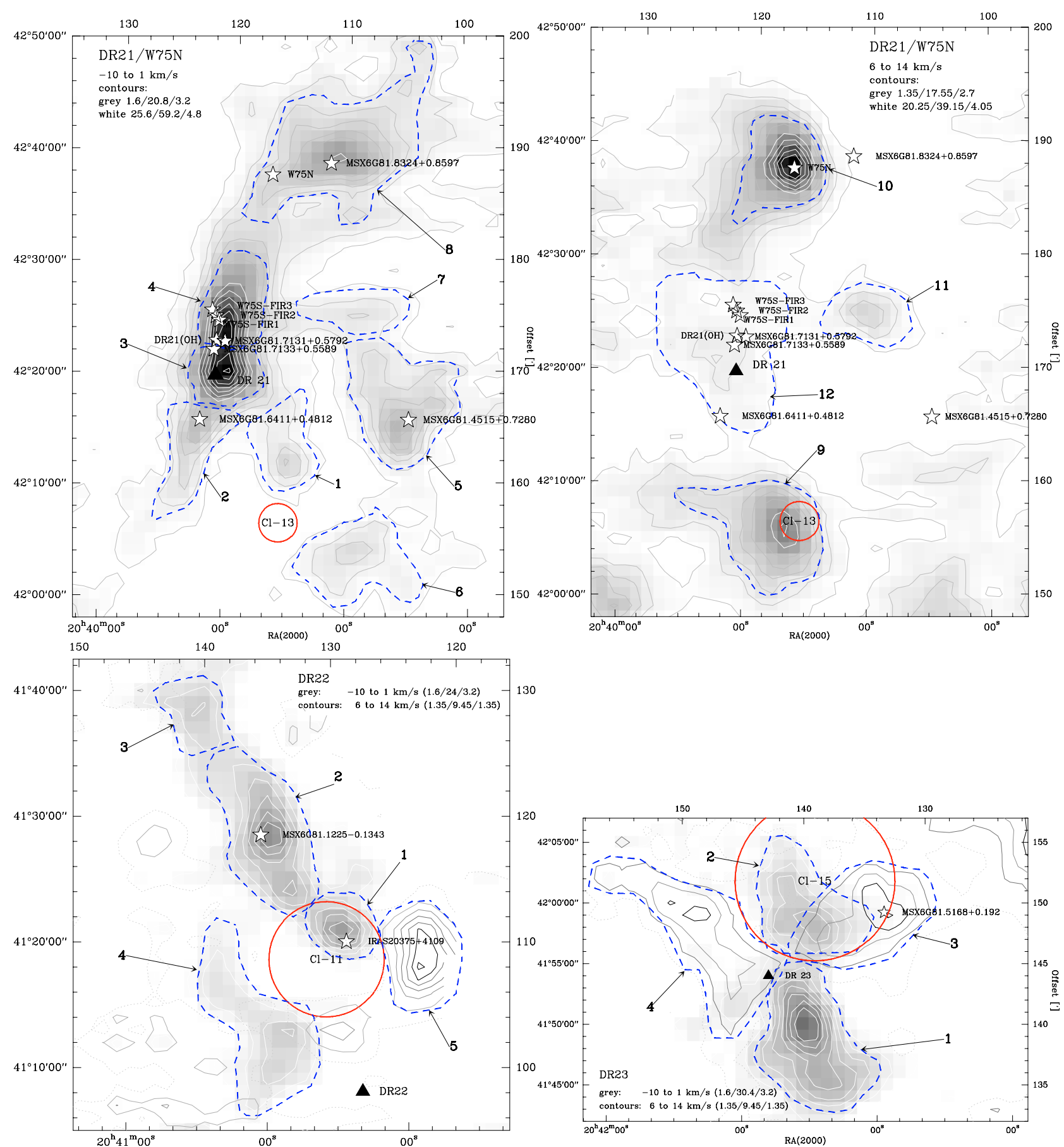

Fig. B.3. The same as Fig. B.1 but for the sources DR21/W75N, DR22, and DR23. Due to confusion, the two main velocity ranges of the CygXNorth region are shown separately.

There are probably several reasons why we do not see clearer trends: first, the OB9 cluster also influences the molecular gas by heating, dissociation, and the dynamical effects of the cluster members. Second, we ignore the 3-dimensional projection of clumps. Even if we conclude that nearly all molecular gas seen in Cygnus X (except for the "Cygnus Rift emission" not considered here) is located at the distance of the OB2 cluster, some complexes are more on the front/back of the complex. Third, we just focus on the largest clumps identified in a $2^{\prime}$ resolution survey. Thus, the properties of the molecular gas is averaged across a rather large volume even though a small-scale structure is present. To overcome this drawback, we use higher angular- resolution (45") FCRAO observations in different line tracers of our Cygnus X survey covering an area of 35 square degrees. Using algorithms that identify smaller entities of gas will help to gain a larger statistic. 
N. Schneider et al.: The Cygnus X region, Online Material $p 7$

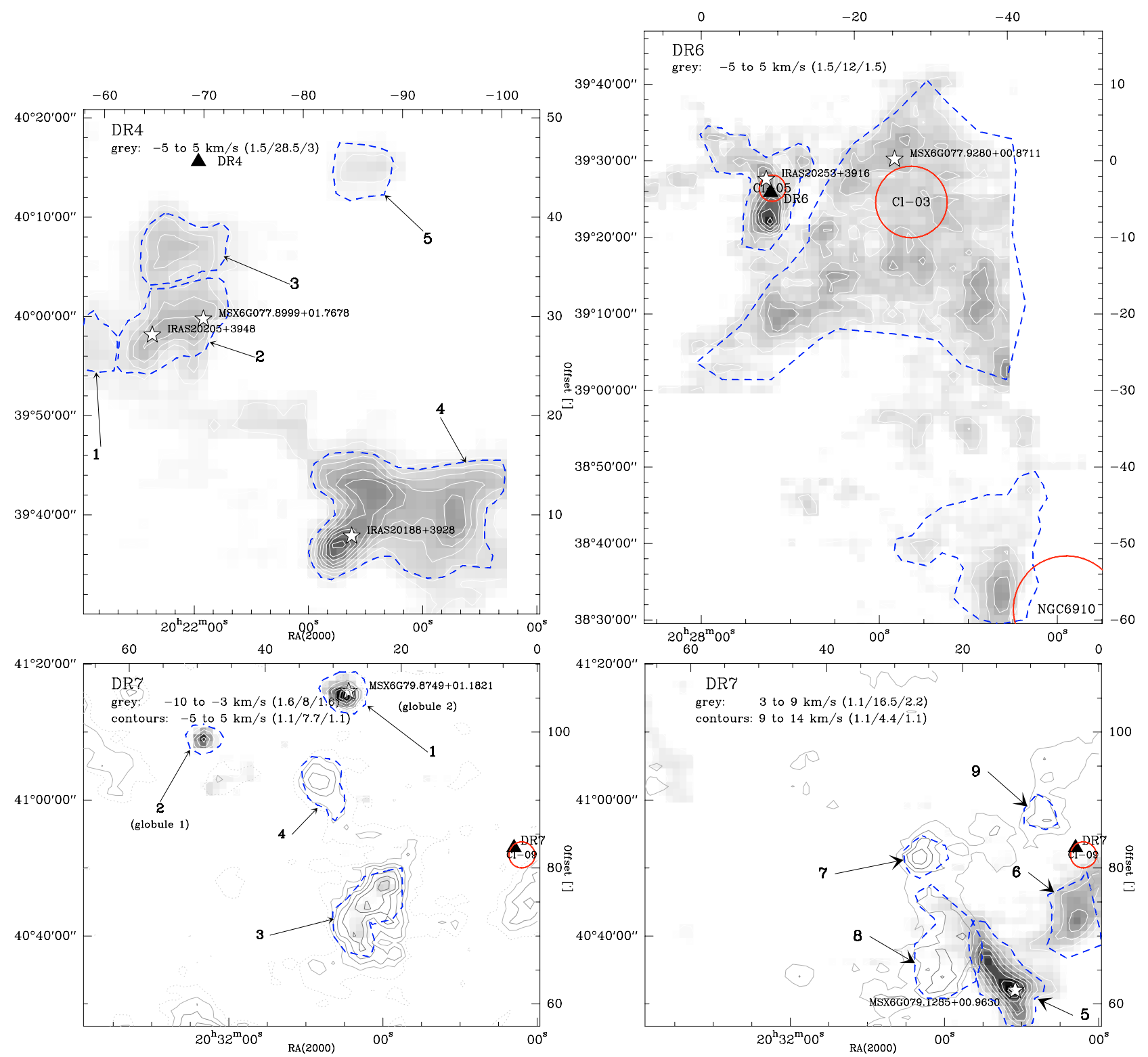

Fig. B.4. The same as Fig. B.1 but for the sources DR4, DR6, and DR7. 
N. Schneider et al.: The Cygnus X region, Online Material $p 8$
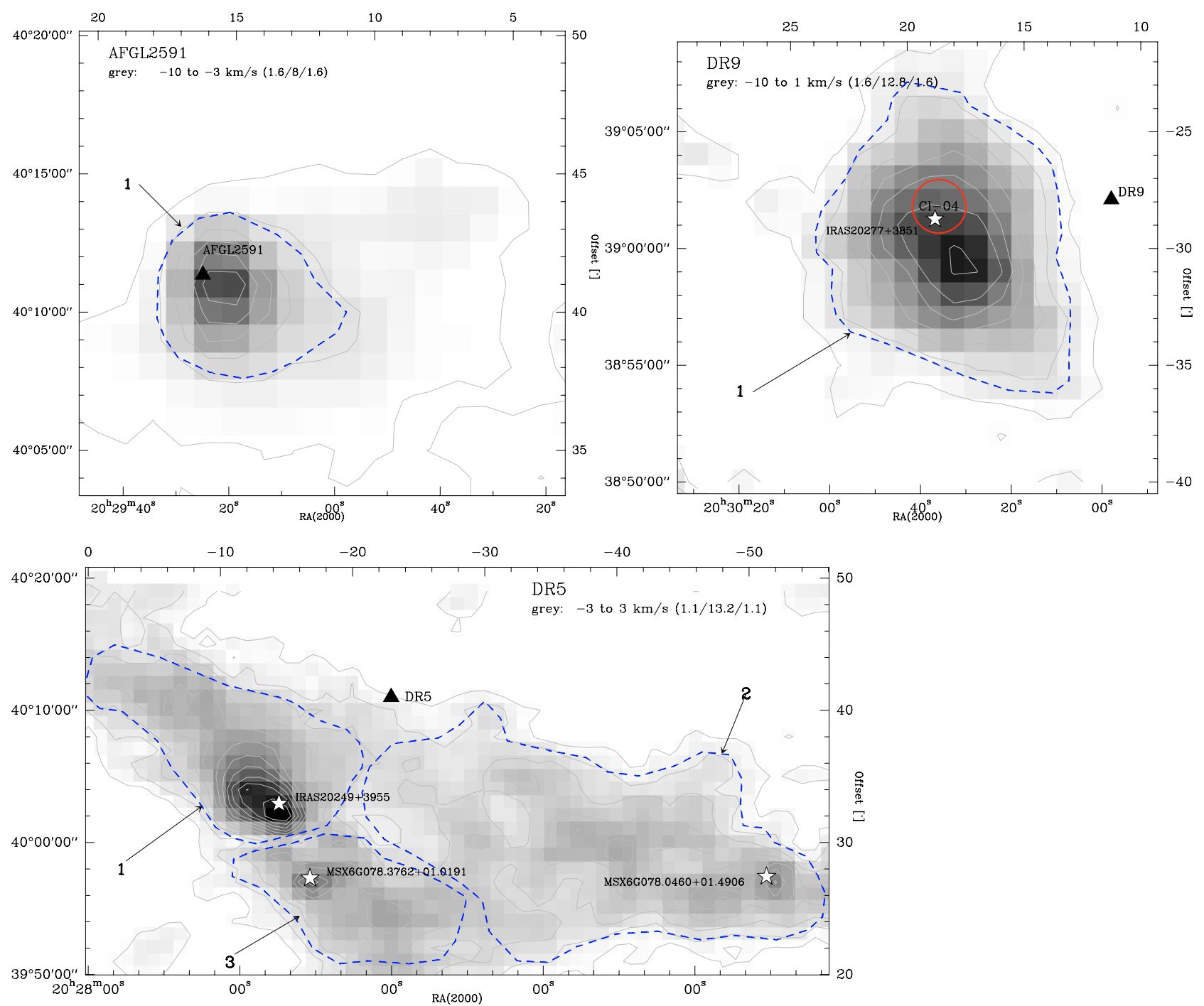

Fig. B.5. The same as Fig. B.1 but for the sources AFGL2591, DR9, and DR5. 
N. Schneider et al.: The Cygnus X region, Online Material $p 9$
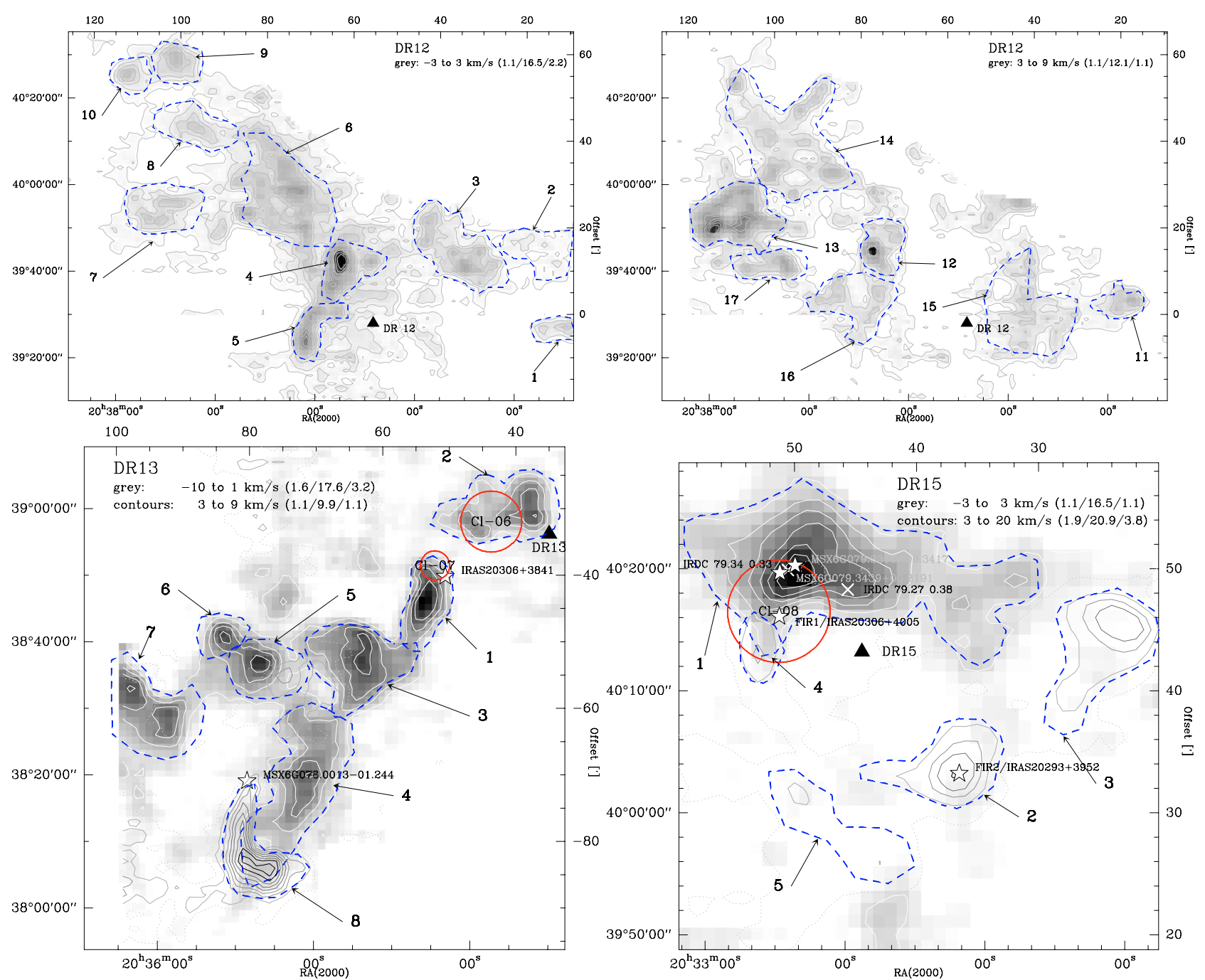

Fig. B.6. The same as Fig. B.1 but for the sources DR12, DR13, and DR15. 


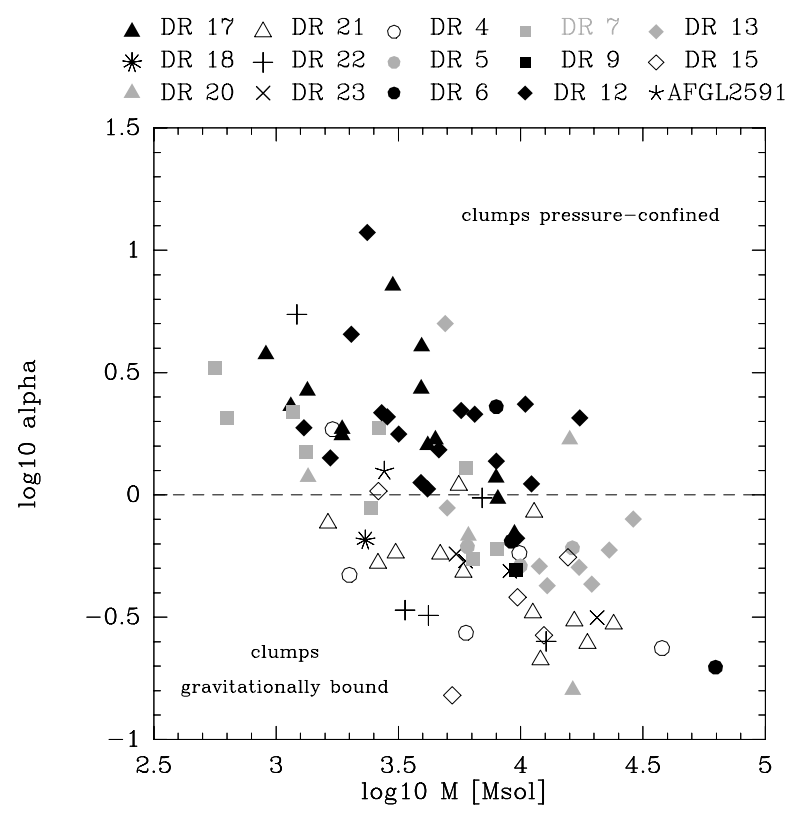

Fig. C.1. Virial parameter $\alpha$ as a function of mass, plotted for the clumps found in Cygnus X. The different symbols allow us to distinguish between clumps of all regions, i.e. DR17-23 in CygX-North and DR4-DR15 and AFGL2591 in CygX-South. Clumps with $\log (\alpha) \simeq 0$ are in gravitational virial equilibrium.
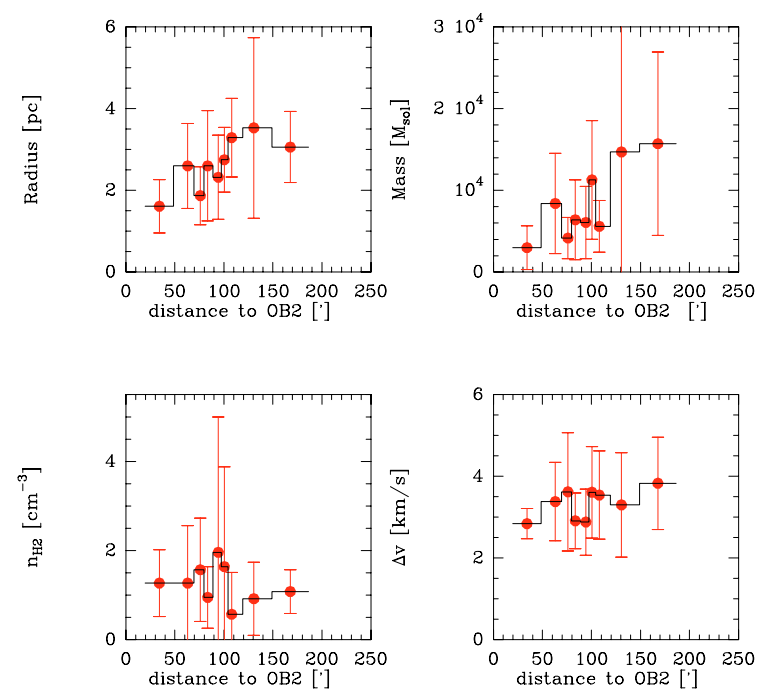

Fig. C.2. Variation in radius, mass, average $\mathrm{H}_{2}$-density $\left(n_{\mathrm{H} 2}\right)$, and line width $(\Delta v)$ of the clumps found in the ${ }^{13} \mathrm{CO}$ Cygnus $\mathrm{X}$ survey with distance to the Cyg OB2 cluster. The data are shown in bins of 10, the error bars indicate standard deviation. 
N. Schneider et al.: The Cygnus X region, Online Material p 11

Table C.1. Physical parameters of the clumps found in the ${ }^{13} \mathrm{CO} 2 \rightarrow 1$ map of the CygX-North region. The columns indicate: (1) the running number of the clump shown in Figs. B.1-B.6; (2) the center velocity of the clump emission derived from the clump averaged ${ }^{13} \mathrm{CO} 2 \rightarrow 1$ spectrum; (3) the line width of the ${ }^{13} \mathrm{CO}$ spectrum averaged across the clump; (4) and (5) ${ }^{12} \mathrm{CO}$ and ${ }^{13} \mathrm{CO} 2 \rightarrow 1$ main beam brightness temperature determined at the position of peak emission; (6) excitation temperature derived from $T_{\mathrm{mb}}\left({ }^{12} \mathrm{CO}\right)$; (7) excitation temperature derived from the ${ }^{13} \mathrm{CO} 3 \rightarrow 2 / 2 \rightarrow 1$ line ratio; (8) opacity calculated from $T_{\mathrm{mb}}\left({ }^{13} \mathrm{CO}\right.$ ) and $T_{\text {ex } 12}$; (9) total ${ }^{13} \mathrm{CO}$ column density; (10) $\mathrm{H}_{2}$ column density; (11) mass of the clump; (12) Equivalent Radius $\left(r=(\operatorname{area} / \pi)^{0.5}\right.$ with the area determined by the polygon), deconvolved with the beamsize; and (13) average $n\left(\mathrm{H}_{2}\right)$ density, assuming a spherical cloud.

\begin{tabular}{|c|c|c|c|c|c|c|c|c|c|c|c|c|}
\hline & $\begin{array}{c}v \\
{\left[\mathrm{~km} \mathrm{~s}^{-1}\right]}\end{array}$ & $\begin{array}{c}\Delta v \\
{\left[\mathrm{~km} \mathrm{~s}^{-1}\right]}\end{array}$ & $\begin{array}{c}T\left({ }^{12} \mathrm{CO}\right) \\
{[\mathrm{K}]}\end{array}$ & $\begin{array}{c}T\left({ }^{13} \mathrm{CO}\right) \\
{[\mathrm{K}]}\end{array}$ & $\begin{array}{c}T_{\text {ex12 }} \\
{[\mathrm{K}]} \\
\end{array}$ & $\begin{array}{c}T_{\mathrm{ex} 13} \\
{[\mathrm{~K}]}\end{array}$ & $\tau$ & $\begin{array}{c}N\left({ }^{13} \mathrm{CO}\right) \\
{\left[10^{16} \mathrm{~cm}^{-2}\right]}\end{array}$ & $\begin{array}{c}N\left(\mathrm{H}_{2}\right) \\
{\left[10^{21} \mathrm{~cm}^{-2}\right]}\end{array}$ & $\begin{array}{l}\text { Mass } \\
{\left[M_{\odot}\right]} \\
\end{array}$ & $\begin{array}{c}\text { Radius } \\
{[\mathrm{pc}]}\end{array}$ & $\begin{array}{c}\left\langle n\left(\mathrm{H}_{2}\right)\right\rangle \\
{\left[10^{3} \mathrm{~cm}^{-3}\right]}\end{array}$ \\
\hline \multicolumn{13}{|c|}{ DR17 } \\
\hline 1 & 14.02 & 3.81 & 14.2 & 4.3 & 19 & 26 & 0.35 & 2.3 & 10.7 & 7939 & 3.07 & 0.56 \\
\hline 2 & 14.46 & 3.76 & 11.3 & 4.1 & 16 & 15 & 0.45 & 2.2 & 10.2 & 1342 & 1.21 & 1.36 \\
\hline 3 & 15.15 & 3.67 & 13.7 & 4.5 & 19 & & 0.39 & 2.0 & 9.3 & 4167 & 2.36 & 0.64 \\
\hline 4 & 16.85 & 2.90 & 10.0 & 2.4 & 15 & & 0.27 & 1.4 & 6.6 & 1857 & 1.85 & 0.58 \\
\hline 5 & 3.81 & 4.12 & 4.0 & 0.5 & 9 & & 0.13 & 2.1 & 10.1 & 908 & 0.96 & 1.71 \\
\hline 6 & 4.41 & 6.53 & 5.8 & 2.2 & 11 & & 0.45 & 1.4 & 6.4 & 3000 & 2.41 & 0.43 \\
\hline \multicolumn{13}{|c|}{$\overline{\mathrm{DR} 17 \mathrm{~N}}$} \\
\hline 1 & 2.50 & 2.84 & 11.1 & 3.0 & 16 & & 0.30 & 1.2 & 5.5 & 1149 & 1.57 & 0.56 \\
\hline 2 & 3.49 & 3.61 & 11.1 & 3.0 & 16 & & 0.30 & 1.6 & 7.3 & 4485 & 2.77 & 0.43 \\
\hline 3 & 3.82 & 3.04 & 11.1 & 3.0 & 16 & & 0.30 & 1.4 & 6.4 & 8068 & 4.02 & 0.26 \\
\hline 4 & 2.60 & 3.77 & 6.0 & 2.0 & 11 & & 0.39 & 0.8 & 3.9 & 3920 & 3.58 & 0.18 \\
\hline 5 & 1.93 & 4.65 & 5.0 & 1.0 & 10 & & 0.22 & 0.9 & 4.0 & 3938 & 3.52 & 0.19 \\
\hline 6 & 4.75 & 2.40 & 5.0 & 1.0 & 10 & & 0.22 & 0.6 & 2.9 & 1862 & 2.87 & 0.16 \\
\hline 7 & 11.43 & 3.50 & 15.5 & 4.1 & 21 & & 0.31 & 3.8 & 18.1 & 9429 & 2.55 & 1.15 \\
\hline \multicolumn{13}{|c|}{ DR18 } \\
\hline 1 & 8.44 & 2.51 & 11.4 & 9.0 & 17 & & 1.50 & 4.1 & 19.3 & 2314 & 1.15 & 2.74 \\
\hline \multicolumn{13}{|l|}{ DR20 } \\
\hline 1 & 3.98 & 2.15 & 9.3 & 6.0 & 14 & 17 & 1.00 & 6.0 & 28.3 & 16313 & 2.69 & 1.71 \\
\hline 2 & -1.32 & 3.34 & 9.4 & 3.6 & 14 & & 0.46 & 4.9 & 23.1 & 6106 & 1.78 & 2.10 \\
\hline 3 & 11.76 & 2.71 & 12.7 & 4.6 & 18 & & 0.44 & 2.8 & 13.2 & 1351 & 1.04 & 2.07 \\
\hline 4 & 12.03 & 5.64 & 9.0 & 2.5 & 14 & & 0.31 & 2.7 & 12.5 & 15860 & 4.02 & 0.51 \\
\hline \multicolumn{13}{|l|}{ DR21 } \\
\hline 1 & -2.03 & 2.33 & 14.5 & 4.5 & 20 & 14 & 0.36 & 3.1 & 14.7 & 3084 & 1.57 & 1.51 \\
\hline 2 & -3.57 & 2.78 & 14.7 & 4.5 & 20 & 14 & 0.36 & 4.9 & 23.1 & 5830 & 1.74 & 2.16 \\
\hline 3 & -2.60 & 4.08 & 22.0 & 10.3 & 27 & 23 & 0.62 & 19.6 & 91.9 & 16559 & 1.45 & 10.3 \\
\hline 4 & -2.99 & 3.62 & 22.0 & 10.7 & 27 & 25 & 0.66 & 16.6 & 78.1 & 18758 & 1.69 & 7.5 \\
\hline 5 & -1.30 & 2.23 & 18.9 & 6.7 & 24 & & 0.42 & 5.3 & 25.1 & 12047 & 2.45 & 1.66 \\
\hline 6 & -1.40 & 2.47 & 13.2 & 4.1 & 18 & 16 & 0.36 & 2.8 & 13.1 & 4686 & 2.10 & 1.00 \\
\hline 7 & -1.68 & 2.09 & 13.3 & 3.8 & 18 & & 0.33 & 2.9 & 13.6 & 2609 & 1.50 & 1.47 \\
\hline 8 & -2.13 & 3.28 & 15.8 & 7.6 & 21 & & 0.65 & 6.4 & 30.3 & 23994 & 3.16 & 1.55 \\
\hline 9 & 8.28 & 2.71 & 24.2 & 8.5 & 30 & 20 & 0.42 & 5.2 & 24.2 & 11206 & 2.40 & 1.64 \\
\hline 10 & 10.30 & 4.91 & 17.0 & 9.5 & 22 & & 0.80 & 8.0 & 37.8 & 11348 & 1.91 & 3.21 \\
\hline 11 & 8.55 & 1.98 & 11.2 & 3.8 & 16 & & 0.40 & 1.7 & 8.2 & 1628 & 1.52 & 0.87 \\
\hline 12 & 7.92 & 3.11 & 3.5 & 1.4 & 8 & & 0.48 & 1.6 & 7.8 & 5582 & 3.02 & 0.42 \\
\hline \multicolumn{13}{|l|}{ DR22 } \\
\hline 1 & -2.91 & 2.28 & 17.4 & 8.2 & 23 & 34 & 0.63 & 6.5 & 30.4 & 4195 & 1.24 & 4.0 \\
\hline 2 & -4.76 & 2.54 & 13.5 & 5.7 & 19 & 17 & 0.53 & 6.0 & 28.3 & 12717 & 2.37 & 1.9 \\
\hline 3 & -5.11 & 2.00 & 10.6 & 4.9 & 16 & 15 & 0.62 & 4.4 & 20.8 & 3369 & 1.36 & 2.5 \\
\hline 4 & -2.92 & 3.66 & 11.7 & 4.7 & 17 & 18 & 0.50 & 3.2 & 14.9 & 6961 & 2.41 & 1.0 \\
\hline 5 & 7.73 & 4.93 & 6.8 & 2.6 & 12 & 15 & 0.45 & 1.7 & 8.0 & 1216 & 1.31 & 1.0 \\
\hline \multicolumn{13}{|l|}{ DR23 } \\
\hline 1 & -5.41 & 3.58 & 20.0 & 8.9 & 25 & & 0.58 & 9.3 & 43.8 & 20529 & 2.41 & 3.0 \\
\hline 2 & -6.87 & 3.12 & 13.7 & 4.3 & 19 & & 0.36 & 5.1 & 23.8 & 9007 & 2.16 & 1.8 \\
\hline 3 & 8.92 & 2.53 & 12.9 & 3.5 & 18 & & 0.31 & 2.6 & 12.4 & 5445 & 2.33 & 0.9 \\
\hline 4 & 9.21 & 2.38 & 13.0 & 3.6 & 18 & & 0.32 & 2.2 & 10.3 & 5942 & 2.69 & 0.6 \\
\hline
\end{tabular}


N. Schneider et al.: The Cygnus X region, Online Material p 12

Table C.2. Physical parameters of the clumps found in the ${ }^{13} \mathrm{CO} 2 \rightarrow 1$ map of the CygX-South region. The columns indicate: (1) the running number of the clump shown in Figs. B.1-B.6; (2) the center velocity of the clump emission derived from the clump averaged ${ }^{13} \mathrm{CO} 2 \rightarrow 1$ spectrum; (3) the line width of the ${ }^{13} \mathrm{CO}$ spectrum averaged across the clump; (4) and (5) ${ }^{12} \mathrm{CO}$ and ${ }^{13} \mathrm{CO} 2 \rightarrow 1$ main beam brightness temperature determined at the position of peak emission, crosses indicate positions where the ${ }^{12} \mathrm{CO} 3 \rightarrow 2$ intensity is shown; (6) excitation temperature derived from $T_{\mathrm{mb}}\left({ }^{12} \mathrm{CO}\right.$ ); (7) excitation temperature derived from the ${ }^{13} \mathrm{CO} 3 \rightarrow 2 / 2 \rightarrow 1$ line ratio; (8) opacity calculated from $T_{\mathrm{mb}}\left({ }^{13} \mathrm{CO}\right)$ and $T_{\text {ex } 12}$; (9) total ${ }^{13} \mathrm{CO}$ column density; (10) $\mathrm{H}_{2}$ column density; (11) mass of the clump; (12) Equivalent Radius $\left(r=(\operatorname{area} / \pi)^{0.5}\right.$ with the area determined by the polygon), deconvolved with the beamsize; and (13) average $n\left(\mathrm{H}_{2}\right)$ density, assuming a spherical cloud.

\begin{tabular}{|c|c|c|c|c|c|c|c|c|c|c|c|c|}
\hline & $\begin{array}{c}v \\
{\left[\mathrm{~km} \mathrm{~s}^{-1}\right]}\end{array}$ & $\begin{array}{c}\Delta v \\
{\left[\mathrm{~km} \mathrm{~s}^{-1}\right]}\end{array}$ & $\begin{array}{c}T\left({ }^{12} \mathrm{CO}\right) \\
{[\mathrm{K}]}\end{array}$ & $\begin{array}{c}T\left({ }^{13} \mathrm{CO}\right) \\
{[\mathrm{K}]}\end{array}$ & $\begin{array}{c}T_{\text {ex12 }} \\
{[\mathrm{K}]}\end{array}$ & $\begin{array}{c}T_{\text {ex13 }} \\
{[\mathrm{K}]}\end{array}$ & $\tau$ & $\begin{array}{c}N\left({ }^{13} \mathrm{CO}\right) \\
{\left[10^{16} \mathrm{~cm}^{-2}\right]}\end{array}$ & $\begin{array}{c}N\left(\mathrm{H}_{2}\right) \\
{\left[10^{21} \mathrm{~cm}^{-2}\right]}\end{array}$ & $\begin{array}{l}\text { Mass } \\
{\left[M_{\odot}\right]}\end{array}$ & $\begin{array}{c}\text { Radius } \\
{[\mathrm{pc}]}\end{array}$ & $\begin{array}{c}\left\langle n\left(\mathrm{H}_{2}\right)\right\rangle \\
{\left[10^{3} \mathrm{~cm}^{-3}\right]}\end{array}$ \\
\hline \multicolumn{13}{|l|}{$\overline{\text { DR4 }}$} \\
\hline 1 & -0.36 & 2.01 & 4.0 & 2.2 & 9 & & 0.79 & 3.7 & 17.5 & 1995 & 1.11 & 2.56 \\
\hline 2 & -2.04 & 3.46 & 13.1 & 4.3 & 18 & & 0.39 & 5.0 & 23.5 & 9882 & 2.28 & 1.67 \\
\hline 3 & -2.05 & 2.03 & 14.8 & 5.2 & 20 & & 0.43 & 4.3 & 20.3 & 5975 & 1.89 & 1.75 \\
\hline 4 & 1.32 & 3.33 & $9.1^{+}$ & 6.1 & 16 & & 0.80 & 6.9 & 32.5 & 37826 & 3.85 & 1.37 \\
\hline 5 & -2.65 & 3.33 & $12.1^{+}$ & 1.6 & 19 & & 0.12 & 2.2 & 10.5 & 1702 & 1.36 & 1.25 \\
\hline \multicolumn{13}{|l|}{ DR5 } \\
\hline 1 & 0.99 & 2.61 & 4.3 & 2.8 & 9 & & 1.00 & 2.1 & 9.9 & 9963 & 3.58 & 0.44 \\
\hline 2 & 0.48 & 2.91 & 4.0 & 2.0 & 9 & & 0.79 & 1.4 & 6.7 & 16269 & 5.57 & 0.10 \\
\hline 3 & 1.35 & 2.45 & 4.0 & 2.0 & 9 & & 0.78 & 1.9 & 8.7 & 6055 & 2.96 & 0.48 \\
\hline \multicolumn{13}{|l|}{ DR6 } \\
\hline 1 & -2.58 & 5.48 & $7.2^{+}$ & 2.2 & 14 & & 0.27 & 2.5 & 11.9 & 7949 & 2.90 & 0.67 \\
\hline 2 & 2.22 & 2.68 & $3.0^{+}$ & 1.4 & 9 & & 0.38 & 2.5 & 12.0 & 62594 & 8.22 & 0.24 \\
\hline 3 & 2.52 & 2.65 & $5.1^{+}$ & 2.8 & 12 & & 0.53 & 1.5 & 7.2 & 9147 & 4.02 & 0.29 \\
\hline \multicolumn{13}{|l|}{ DR7 } \\
\hline 1 & -4.37 & 2.85 & $16.2^{+}$ & 3.45 & 24 & & 0.21 & 1.3 & 6.1 & 1170 & 1.50 & 0.66 \\
\hline 2 & -10.75 & 2.83 & $5.6^{+}$ & 2.6 & 12 & & 0.44 & 1.1 & 5.0 & 564 & 1.11 & 0.72 \\
\hline 3 & -2.56 & 3.04 & $3.6^{+}$ & 1.2 & 10 & & 0.27 & 3.4 & 16.2 & 7967 & 2.48 & 1.07 \\
\hline 4 & -1.77 & 2.45 & $3.2^{+}$ & 1.5 & 9 & & 0.39 & 2.1 & 9.9 & 2447 & 1.72 & 0.94 \\
\hline 5 & 7.03 & 2.49 & $20.2^{+}$ & 6.0 & 28 & & 0.31 & 2.3 & 11.1 & 6374 & 2.69 & 0.67 \\
\hline 6 & 5.58 & 3.23 & $10.0^{+}$ & 3.7 & 17 & & 0.37 & 1.4 & 6.4 & 2628 & 2.25 & 0.47 \\
\hline 7 & 11.56 & 2.62 & $5.8^{+}$ & 2.2 & 12 & & 0.34 & 1.7 & 7.9 & 1331 & 1.39 & 0.92 \\
\hline 8 & 11.98 & 3.53 & $2.5^{+}$ & 0.7 & 8 & & 0.20 & 1.8 & 8.6 & 6000 & 2.96 & 0.47 \\
\hline 9 & 13.15 & 2.49 & $5.6^{+}$ & 1.3 & 12 & & 0.19 & 1.4 & 6.6 & 631 & 1.00 & 1.07 \\
\hline \multicolumn{13}{|l|}{ DR9 } \\
\hline 1 & -2.88 & 2.86 & 11.9 & 4.4 & 17 & & 0.44 & 3.4 & 15.8 & 9561 & 2.76 & 0.93 \\
\hline \multicolumn{13}{|c|}{ DR12 } \\
\hline 1 & 0.49 & 2.46 & 4.7 & 1.4 & 9 & & 0.34 & 0.9 & 4.2 & 1300 & 1.93 & 0.38 \\
\hline 2 & -1.96 & 6.35 & 4.7 & 1.4 & 9 & & 0.47 & 0.6 & 2.8 & 2358 & 3.30 & 0.13 \\
\hline 3 & -1.78 & 2.58 & 4.6 & 1.8 & 9 & & 0.45 & 1.2 & 5.9 & 9622 & 4.59 & 0.21 \\
\hline 4 & -0.20 & 4.07 & $6.0^{+}$ & 4.8 & 13 & & 0.96 & 2.1 & 10.1 & 7952 & 3.15 & 0.52 \\
\hline 5 & -1.45 & 2.88 & $13.1^{+}$ & 3.9 & 20 & & 0.29 & 1.7 & 8.1 & 4165 & 2.54 & 0.52 \\
\hline 6 & -1.34 & 5.61 & $4.0^{+}$ & 2.1 & 10 & & 0.47 & 1.6 & 7.5 & 17425 & 5.46 & 0.22 \\
\hline 7 & 3.27 & 4.50 & $4.5^{+}$ & 1.9 & 11 & & 0.37 & 0.9 & 4.4 & 4634 & 3.67 & 0.19 \\
\hline 8 & -0.69 & 4.26 & $1.5^{+}$ & 0.7 & 7 & & 0.32 & 1.4 & 6.5 & 5712 & 3.33 & 0.32 \\
\hline 9 & 1.63 & 3.28 & $5.8^{+}$ & 1.7 & 12 & & 0.25 & 1.1 & 5.1 & 2853 & 2.64 & 0.31 \\
\hline 10 & 0.78 & 4.42 & $2.9^{+}$ & 1.5 & 9 & & 0.43 & 1.1 & 5.0 & 2030 & 2.25 & 0.36 \\
\hline 11 & 8.95 & 2.26 & $5.0^{+}$ & 1.1 & 11 & & 0.18 & 0.9 & 4.2 & 1667 & 2.21 & 0.31 \\
\hline 12 & 5.64 & 3.08 & $12.5^{+}$ & 4.3 & 20 & & 0.35 & 1.1 & 5.0 & 3171 & 2.83 & 0.29 \\
\hline 13 & 4.42 & 5.18 & $4.8^{+}$ & 3.0 & 11 & & 0.62 & 1.5 & 7.0 & 10446 & 4.37 & 0.26 \\
\hline 14 & 6.81 & 3.06 & $4.4^{+}$ & 1.2 & 11 & & 0.22 & 0.8 & 3.6 & 11072 & 6.27 & 0.09 \\
\hline 15 & 7.70 & 3.64 & $4.1^{+}$ & 1.4 & 10 & & 0.28 & 0.7 & 3.4 & 6490 & 5.00 & 0.11 \\
\hline 16 & 4.13 & 2.34 & $5.5^{+}$ & 1.7 & 12 & & 0.27 & 0.7 & 3.4 & 3909 & 3.83 & 0.14 \\
\hline 17 & 4.62 & 3.19 & $4.2^{+}$ & 2.2 & 10 & & 0.48 & 1.0 & 5.0 & 2700 & 2.75 & 0.27 \\
\hline \multicolumn{13}{|c|}{ DR13 } \\
\hline 1 & -0.29 & 3.30 & 16.5 & 5.6 & 22 & & 0.41 & 5.9 & 27.8 & 12850 & 2.40 & 1.88 \\
\hline 2 & -0.12 & 3.45 & 5.5 & 3.7 & 10 & & 1.07 & 4.6 & 21.7 & 19520 & 3.38 & 1.04 \\
\hline 3 & 0.21 & 4.46 & $12.0^{+}$ & 4.7 & 19 & & 0.41 & 5.7 & 27.0 & 23017 & 3.29 & 1.33 \\
\hline 4 & -0.72 & 5.06 & $8.3^{+}$ & 3.5 & 15 & & 0.42 & 4.3 & 20.0 & 28841 & 4.29 & 0.76 \\
\hline 5 & -1.025 & 3.38 & $9.65^{+}$ & 3.0 & 17 & & 0.30 & 4.9 & 22.8 & 11924 & 2.55 & 1.45 \\
\hline 6 & -2.78 & 3.56 & $9.65^{+}$ & 3.0 & 17 & & 0.30 & 4.5 & 21.4 & 5011 & 1.67 & 2.08 \\
\hline 7 & -1.75 & 3.63 & $11.2^{+}$ & 3.8 & 18 & & 0.34 & 4.6 & 21.7 & 17330 & 3.18 & 1.11 \\
\hline 8 & 1.75 & 6.33 & $8.5^{+}$ & 4.6 & 15 & & 0.57 & 1.5 & 7.2 & 4916 & 2.94 & 0.40 \\
\hline
\end{tabular}


N. Schneider et al.: The Cygnus X region, Online Material p 13

Table C.2. continued.

\begin{tabular}{|c|c|c|c|c|c|c|c|c|c|c|c|c|}
\hline & $\begin{array}{c}v \\
{\left[\mathrm{~km} \mathrm{~s}^{-1}\right]}\end{array}$ & $\begin{array}{c}\Delta v \\
{\left[\mathrm{~km} \mathrm{~s}^{-1}\right]}\end{array}$ & $\begin{array}{c}T\left({ }^{12} \mathrm{CO}\right) \\
{[\mathrm{K}]}\end{array}$ & $\begin{array}{c}T\left({ }^{13} \mathrm{CO}\right) \\
{[\mathrm{K}]}\end{array}$ & $\begin{array}{c}T_{\text {ex12 }} \\
{[\mathrm{K}]}\end{array}$ & $\begin{array}{c}T_{\text {ex13 }} \\
{[\mathrm{K}]}\end{array}$ & $\tau$ & $\begin{array}{c}N\left({ }^{13} \mathrm{CO}\right) \\
{\left[10^{16} \mathrm{~cm}^{-2}\right]}\end{array}$ & $\begin{array}{c}N\left(\mathrm{H}_{2}\right) \\
{\left[10^{21} \mathrm{~cm}^{-2}\right]}\end{array}$ & $\begin{array}{l}\text { Mass } \\
{\left[M_{\odot}\right]}\end{array}$ & $\begin{array}{c}\text { Radius } \\
{[\mathrm{pc}]}\end{array}$ & $\begin{array}{c}\left\langle n\left(\mathrm{H}_{2}\right)\right\rangle \\
{\left[10^{3} \mathrm{~cm}^{-3}\right]}\end{array}$ \\
\hline \multicolumn{13}{|l|}{ DR15 } \\
\hline 1 & 0.47 & 3.13 & 6.4 & 3.8 & 11 & & 0.87 & 2.4 & 11.2 & 15605 & 4.23 & 0.43 \\
\hline 2 & 5.57 & 3.06 & 10.0 & 5.6 & 15 & & 0.81 & 7.0 & 33.0 & 9708 & 1.89 & 2.84 \\
\hline 3 & 6.72 & 2.68 & 15.5 & 6.0 & 21 & & 0.48 & 6.7 & 31.4 & 12448 & 2.21 & 2.31 \\
\hline 4 & 3.74 & 3.60 & 9.8 & 3.7 & 15 & & 0.47 & 5.8 & 27.3 & 2618 & 1.00 & 4.42 \\
\hline 5 & 7.55 & 1.47 & 5.0 & 2.2 & 10 & & 0.55 & 4.3 & 20.3 & 5246 & 1.76 & 1.87 \\
\hline \multicolumn{13}{|l|}{ AFGL2591 } \\
\hline 1 & -6.07 & 3.45 & 6.9 & 4.9 & 12 & & 1.19 & 3.5 & 16.5 & 2768 & 1.39 & 1.92 \\
\hline
\end{tabular}

